\title{
QUASI-CLASSICAL ASYMPTOTICS FOR FUNCTIONS OF WIENER-HOPF OPERATORS: SMOOTH VERSUS NON-SMOOTH SYMBOLS
}

\author{
Alexander V. Sobolev
}

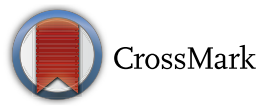

\begin{abstract}
We consider functions of Wiener-Hopf type operators on the Hilbert space $\mathrm{L}^{2}\left(\mathbb{R}^{d}\right)$. It has been known for a long time that the quasi-classical asymptotics for traces of resulting operators strongly depend on the smoothness of the symbol: for smooth symbols the expansion is power-like, whereas discontinuous symbols (e.g. indicator functions) produce an extra logarithmic factor. We investigate the transition regime by studying symbols depending on an extra parameter $T \geq 0$ in such a way that the symbol tends to a discontinuous one as $T \rightarrow 0$. The main result is two-parameter asymptotics (in the quasi-classical parameter and in $T$ ), describing a transition from the smooth case to the discontinuous one. The obtained asymptotic formulas are used to analyse the low-temperature scaling limit of the spatially bipartite entanglement entropy of thermal equilibrium states of non-interacting fermions.
\end{abstract}

\section{Contents}

1 Introduction . . . . . . . . . . . . . . . . . . . . . 677

2 Preliminaries: main result . . . . . . . . . . . . . . . . . . . . 681

2.1 Schatten-von Neumann classes. . . . . . . . . . . . . . . . . . 681

2.2 The domains and regions. . . . . . . . . . . . . . . . . . 683

2.3 The main result. . . . . . . . . . . . . . . . . . . 683

2.4 Anti-Wick quantization. . . . . . . . . . . . . . . . . 686

3 The Fermi symbol: entanglement entropy and local entropy . . . . . . . . . . . . . 687

4 Smooth functions of self-adjoint operators . . . . . . . . . . . . . . . . . . 690

4.1 The Helffer-Sjöstrand formula. . . . . . . . . . . . . . . . . . . . . . . . 690

4.2 Quasi-commutators. . . . . . . . . . . . . . . . . 691

4.3 Elementary estimates for pseudo-differential operators. . . . . . . . . . . . 693

5 Estimates for Wiener-Hopf operators . . . . . . . . . . . . . . . . . . . . 694

5.1 Some basic bounds. . . . . . . . . . . . . . . . . . . . . . . . . 694

5.2 Bounds for more general operators. . . . . . . . . . . . . . . . . 696

5.3 Multi-scale symbols. . . . . . . . . . . . . . . . . 697

6 Bounds involving the symbol $a_{T}$ : Proof of Theorem $2.6 \ldots \ldots \ldots$

6.1 Elementary bounds for the symbol $a_{T} \ldots \ldots \ldots \ldots$. . . . . . 698

Keywords and phrases: Non-smooth functions of Wiener-Hopf operators, Asymptotic trace formulas, Entanglement entropy

Mathematics Subject Classification: Primary 47G30, 35S05; Secondary 45M05, 47B10, 47B35 
6.2 Bounds for pseudo-differential operators with $a_{T} \ldots \ldots \ldots \ldots$. . . . . 700

6.3 Lattice norm bounds for pseudo-differential operators. . . . . . . . . . . . . 701

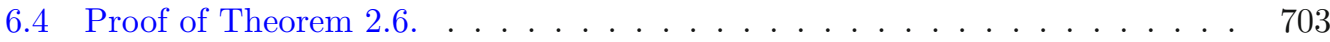

7 Asymptotics for discontinuous symbols . . . . . . . . . . . . . . . 704

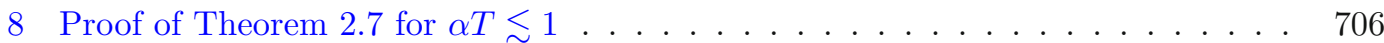

8.1 Localization estimates for the operator $D_{\alpha}(a, \Lambda ; f) \ldots \ldots \ldots \ldots$

8.2 Proof of Theorem 2.7 for $\alpha T \lesssim 1 \ldots \ldots \ldots \ldots$. . . . . . . . . 708

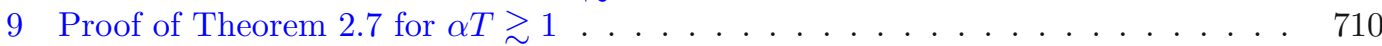

9.1 Proof of Theorem 2.7: basic smooth domains $\Lambda \ldots \ldots \ldots$. . . . . . . 710

9.2 Proof of Theorem 2.7: basic piece-wise smooth domains $\Lambda$. . . . . . . . . 712

9.3 Proof of Theorem 2.7: completion. . . . . . . . . . . . . . . 714

10 Comparison with known asymptotic formulas . . . . . . . . . . . . . . 714

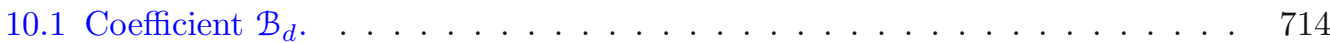

10.2 Coefficient $\mathcal{B}_{d}$ for the symbol $a_{T} \ldots \ldots \ldots \ldots \ldots \ldots \ldots$

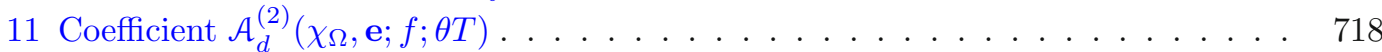

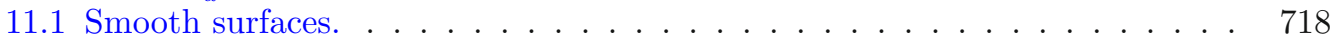

11.2 Function $m \ldots \ldots \ldots \ldots \ldots$

11.3 Asymptotics of $\mathcal{A}_{d}^{(2)}\left(\chi_{\Omega}, \mathbf{e} ; f ; \theta T\right) \ldots \ldots \ldots \ldots \ldots \ldots$

11.4 Proof of Theorem 10.1. . . . . . . . . . . . . . . . . . . . . 723

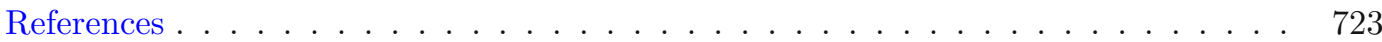

\section{Introduction}

The present paper is devoted to the study of (bounded, self-adjoint) operators of the form

$$
W_{\alpha}:=W_{\alpha}(a ; \Lambda):=\chi_{\Lambda} \mathrm{Op}_{\alpha}(a) \chi_{\Lambda}, \alpha>0
$$

on $\mathrm{L}^{2}\left(\mathbb{R}^{d}\right), d \geq 1$, where $\chi_{\Lambda}$ is the indicator function of a set $\Lambda \subset \mathbb{R}^{d}$. The notation $\mathrm{Op}_{\alpha}(a)$ stands for the $\alpha$-pseudo-differential operator with symbol $a=a(\boldsymbol{\xi})$, which acts on Schwartz functions $u$ on $\mathbb{R}^{d}$ as

$$
\left(\mathrm{Op}_{\alpha}(a) u\right)(\mathbf{x}):=\frac{\alpha^{d}}{(2 \pi)^{d}} \iint e^{i \alpha \boldsymbol{\xi} \cdot(\mathbf{x}-\mathbf{y})} a(\boldsymbol{\xi}) u(\mathbf{y}) d \mathbf{y} d \boldsymbol{\xi}, \quad \mathbf{x} \in \mathbb{R}^{d} .
$$

Integrals without indication of the integration domain always mean integration over $\mathbb{R}^{d}$ with the value of $d$ which is clear from the context. We call the operator (1.1) a (truncated) Wiener-Hopf operator. We are interested in the asymptotics of the trace of the following operator difference

$$
D_{\alpha}(a, \Lambda ; f):=\chi_{\Lambda} f\left(W_{\alpha}(a ; \Lambda)\right) \chi_{\Lambda}-W_{\alpha}(f \circ a ; \Lambda),
$$

as $\alpha \rightarrow \infty$, with some suitably chosen functions $f$. The reciprocal parameter $\alpha^{-1}$ can be interpreted as Planck's constant, and hence the limit $\alpha \rightarrow \infty$ can be regarded as the quasi-classical limit. Sometimes a different point of view is convenient: by changing the variables one easily sees that the operator (1.2) is unitarily equivalent 
to $D_{1}(a, \alpha \Lambda ; f)$, so that the asymptotics $\alpha \rightarrow \infty$ can be interpreted as a large-scale limit.

The second operator on the right-hand side of (1.2) can be viewed as a regularizing term: it makes the operator (1.2) trace class even if $f(0) \neq 0$ and $\Lambda$ is unbounded, see Condition 2.4 for precise assumptions on $\Lambda$. On the other hand, if $f(0)=0, \Lambda$ is bounded and the symbol $a$ decays fast at infinity, then $W_{\alpha}(f \circ a ; \Lambda)$ is trace class itself and an elementary calculation shows that

$$
\operatorname{tr} W_{\alpha}(f \circ a ; \Lambda)=\frac{\alpha^{d}}{(2 \pi)^{d}}|\Lambda| \int f(a(\boldsymbol{\xi})) d \boldsymbol{\xi},
$$

where $|\Lambda|$ is the $d$-dimensional Lebesgue measure of $\Lambda$.

Asymptotic properties of $D_{\alpha}(a, \Lambda ; f)$ depend strongly on the smoothness of the symbol $a$. For smooth symbols $a$, smooth functions $f$ and smooth bounded regions $\Lambda$, the full asymptotic expansion of $\operatorname{tr} D_{\alpha}(a, \Lambda ; f)$ in powers of $\alpha^{-1}$ was derived by Budylin-Buslaev [BB91] and H. Widom [Wid85], and we refer to these papers for the history of the problem and further references. We are concerned only with the leading term asymptotics: they have the form

$$
\operatorname{tr} D_{\alpha}(a, \Lambda ; f)=\alpha^{d-1} \mathcal{B}_{d}(a)+O\left(\alpha^{d-2}\right), \alpha \rightarrow \infty,
$$

where the coefficient $\mathcal{B}_{d}(a)=\mathcal{B}_{d}(a ; \partial \Lambda, f)$ is defined in (10.5).

For symbols $a$ with jump discontinuities the asymptotics have a different form. For instance, for $a=\chi_{\Omega}$ with a bounded piece-wise region $\Omega$, it was found that

$$
\operatorname{tr} D_{\alpha}\left(\chi_{\Omega}, \Lambda ; f\right)=U \mathfrak{V}_{1} \alpha^{d-1} \log \alpha+o\left(\alpha^{d-1} \log \alpha\right), \alpha \rightarrow \infty,
$$

for a bounded region $\Lambda \subset \mathbb{R}^{d}$, with explicitly given coefficients $U=U(f)$ and $\mathfrak{V}_{1}=\mathfrak{V}_{1}(1, \partial \Lambda, \partial \Omega)$, see (2.11) and (2.12) for the definitions. Discontinuous symbols came into prominence after the papers by M. E. Fisher and R. E. Hartwig, see e.g. [FH98], on determinants of truncated Toeplitz matrices. Ever since, discontinuity of the symbol is sometimes referred to as one of the two Fisher-Hartwig singularities. The formula (1.5) for smooth functions $f$ was proved by Landau-Widom [LW80], Widom [Wid82b] (for $d=1$ ) and by A.V. Sobolev [Sob13,Sob15] (for arbitrary $d \geq 1$ ). These issues have been exhaustively studied for the Toeplitz matrices, see e.g. survey [Kra11] for references.

In the present paper we study the transition from the smooth to discontinuous symbols. Precisely, we consider smooth symbols $a=a_{T}$ depending on the additional parameter $T>0$, in such a way that $a_{T}(\boldsymbol{\xi}) \rightarrow \chi_{\Omega}(\boldsymbol{\xi})$, as $T \rightarrow 0$ pointwise, with a region $\Omega \subset \mathbb{R}^{d}$, and satisfying some mild regularity conditions, see (2.8), (2.9). The objective is to investigate the asymptotics of $\operatorname{tr} D_{\alpha}\left(a_{T}, \Lambda ; f\right)$ as $T \rightarrow 0$ and $\alpha \rightarrow \infty$ simultaneously and independently. The sharp bounds for this quantity are stated in Theorem 2.6, and the asymptotic results are collected in Theorem 2.7. The function $f$ is not assumed to be globally smooth, but is allowed to have finitely many 
points of non-smoothness. A typical example of such a function, with one point of non-smoothness, is

$$
f(t)=|t-z|^{\gamma}, t \in \mathbb{R}
$$

with some fixed $z \in \mathbb{R}$, where $\gamma>0$. The inclusion of non-smooth functions $f$ is far from trivial, but the relevant tools have been developed earlier (see [Sob,LSS16b]), and we use them with minor modifications.

The study of such a two-parameter behaviour seems to be an interesting natural problem of asymptotic analysis in its own right. Our motivation however comes from the analysis of large-scale behaviour of the spatially bipartite entanglement entropy of free fermions in thermal equilibrium. This question amounts to studying the trace of the operator (1.2) with a specific choice of the symbol $a_{T}$ and function $f$. The symbol is taken to be the Fermi symbol

$$
a_{T}(\boldsymbol{\xi}):=a_{T, \mu}(\boldsymbol{\xi}):=\frac{1}{1+\exp \frac{h(\boldsymbol{\xi})-\mu}{T}}, \quad \boldsymbol{\xi} \in \mathbb{R}^{d},
$$

where $T>0$ is the temperature, and $\mu \in \mathbb{R}$ is the chemical potential. The function $h=h(\boldsymbol{\xi})$ is the free Hamiltonian and we assume that $h(\boldsymbol{\xi}) \rightarrow \infty$ as $|\boldsymbol{\xi}| \rightarrow \infty$, so that the Fermi sea $\Omega=\left\{\boldsymbol{\xi} \in \mathbb{R}^{d}: h(\boldsymbol{\xi})<\mu\right\}$ is a bounded set. Note that $a_{T, \mu} \rightarrow \chi_{\Omega}, T \rightarrow 0$, pointwise.

The function(1.7) is a typical representative of the symbols $a_{T}$ featuring in Theorem 2.7, so for the sake of discussion in this introduction, we assume that $a_{T}$ is simply given by the symbol (1.7). The form of the asymptotics in the main theorem depends on the relation between $\alpha$ and $T$, the regime $\alpha T=$ const being the critical one. If $\alpha T \leq$ const, then the asymptotics have exactly the form (1.5), i.e. the same as in the case $a=\chi_{\Omega}$. If however $\alpha T \geq$ const, then

$$
\operatorname{tr} D_{\alpha}\left(a_{T}, \Lambda ; f\right)=U \mathfrak{V}_{1} \alpha^{d-1} \log \frac{1}{T}+o\left(\alpha^{d-1} \log \frac{1}{T}\right), T \rightarrow 0 .
$$

As proved in Theorem 10.1, the asymptotic formula (1.8) can be recast in the form (1.4) as follows:

$$
\operatorname{tr} D_{\alpha}\left(a_{T}, \Lambda ; f\right)=\alpha^{d-1} \mathcal{B}_{d}\left(a_{T}\right)+o\left(\alpha^{d-1} \log \frac{1}{T}\right), T \rightarrow 0, \alpha T \geq \text { const. }
$$

Therefore the asymptotic results in Theorem 2.7 do indeed bridge the dichotomy between smooth and discontinuous symbols.

Returning to the large-scale asymptotics of the entanglement entropy, they follow from Theorem 2.7 with the symbol (1.7), and with the function $f$ which is chosen to be one of the $\gamma$-Rényi entropy functions $\eta_{\gamma}, \gamma>0$, that are defined in (3.5) and (3.6). Thus our results provide low-temperature scaling limit of the entanglement entropy in all dimensions $d \geq 2$. These formulas were announced in the article [LSS16a] without underlying mathematical details. The case of zero temperature, i.e. that of 
$a=\chi_{\Omega}$ was studied in [LSS]. The two-parameter asymptotics for the entropy were obtained in [LSS16b] for $d=1$. The formulas found there hold for $\alpha \rightarrow \infty$ and $\alpha T \geq$ const. In particular, these conditions allow the limit $\alpha \rightarrow \infty, T=$ const. On the other hand, Theorem 2.7 always requires $T \rightarrow 0$, but allows $\alpha T \leq$ const.

The idea is to prove the main result for smooth functions $f$ first. In the case $\alpha T \leq$ const an elementary argument allows us to replace the symbol $a_{T}$ by its limit $\chi_{\Omega}$, and subsequently use the known asymptotic results for discontinuous symbols, see [Sob13,Sob15]. This produces a formula of the form (1.5). The case $\alpha T \geq$ const is substantially more difficult. Here we observe that different parts of the region $\Lambda$ give different contribution to the trace asymptotics. Namely, the boundary layer of width $(\alpha T)^{-1}$ gives the main input into the answer. This input is found again by replacing the symbol $a_{T}$ with the function $\chi_{\Omega}$, and using the results of [Sob13, Sob15]. However, in contrast to the $\alpha T \leq$ const case, due to the small size of the boundary layer, the resulting asymptotic formula contains $\log \frac{1}{T}$ instead of $\log \alpha$. The extension of these results to non-smooth functions $f$ follows the classical idea of asymptotic analysis: we approximate $f$ by smooth functions, and for the error we use bounds for the trace norm of (1.2) that explicitly depend on the function $f$ and the parameter $T$. In the abstract setting such bounds had been proved in [Sob], and later they were used for pseudo-differential operators in [LSS16b] for the case $\alpha T \geq$ const.

The first principal technical ingredient is estimates for pseudo-differential operators in the Schatten-von Neumann classes $\mathfrak{S}_{q}, q>0$. Since the symbol $a_{T}$ depends on the extra parameter $T$, the main effort goes into controlling the dependence of the estimates on the symbol, or at least on the parameter $T$. Here we rely mostly on the bounds obtained in [Sob14] and [LSS16b], but also derive some new ones, see e.g. (5.11). Although the main results are concerned with traces and trace norms, one should also stress that some intermediate results require bounds in the classes $\mathfrak{S}_{q}$ with $q \in(0,1)$. The need for this becomes transparent if in the operator (1.2) one takes, as an example, the function (1.6) with $0<\gamma<1$.

The second ingredient is the trace asymptotics for the operator (1.2) with a discontinuous symbol of the type $a=\chi_{\Omega}$. As mentioned earlier, these were obtained in [Sob13,Sob15]. Again, it is crucial that these results are uniform in the region $\Lambda$ in some suitable sense.

Different parts of the proof have different degree of detail. In maximal detail we present new arguments, in particular those involving explicit control of the dependence on the parameter $T$. At the same time, the parts of reasoning that repeat previously known proofs in new circumstances, are just sketched and sometimes, omitted.

The plan of the paper is as follows: in Section 2 we provide some basic information on Schatten-von Neumann classes, including the useful $q$-triangle inequality (2.1), and state the main results, see Theorems 2.6 and 2.7. The whole of Section 3 is devoted to applications of the main theorems to the study of various entropies of fermionic systems. Some elementary estimates for smooth functions of self-adjoint 
operators are presented in Section 4. In Sections 5 and 6 we collect the necessary Schatten-von Neumann estimates for pseudo-differential operators and Wiener-Hopf operators, and prove Theorem 2.6. Section 7 contains preliminary information about trace asymptotics for discontinuous symbols. The main theorems are proved in Sections 8 and 9. Rewriting the results for $\alpha T \gtrsim 1$ in the form (1.9) takes a lot of technical work, which is done in Sections 10 and 11.

Throughout the paper we adopt the following convention. For two non-negative numbers (or functions) $X$ and $Y$ depending on some parameters, we write $X \lesssim Y$ (or $Y \gtrsim X)$ if $X \leq C Y$ with some positive constant $C$ independent of those parameters. If $X \lesssim Y$ and $X \gtrsim Y$, then we write $X \asymp Y$. For example, $\alpha T \asymp 1$ means that $c \leq \alpha T \leq C$ with some constants $C$, $c$, independent of $\alpha$ and $T$. To avoid confusion we often make explicit comments on the nature of (implicit) constants in the bounds.

The notation $B(\mathbf{z}, R) \subset \mathbb{R}^{d}, \mathbf{z} \in \mathbb{R}^{d}, R>0$, is used for the open ball of radius $R$, centred at the point $\mathbf{z}$.

For any vector $\mathbf{v} \in \mathbb{R}^{n}, n=1,2, \ldots$ we use the standard notation $\langle\mathbf{v}\rangle=$ $\sqrt{1+|\mathbf{v}|^{2}}$.

\section{Preliminaries: main result}

2.1 Schatten-von Neumann classes. We use some well-known facts about Schatten-von Neumann operator ideals $\mathfrak{S}_{q}, q>0$. Detailed information on these ideals can be found e.g. in [BS87, GK69,Pie78, Sim05]. We shall point out only some basic facts. For a compact operator $A$ on a separable Hilbert space $\mathcal{H}$ denote by $s_{n}(A), n=1,2, \ldots$ its singular values, i.e., the eigenvalues of the operator $|A|:=$ $\sqrt{A^{*} A}$. We denote the identity operator on $\mathcal{H}$ by $I$. The Schatten-von Neumann ideal $\mathfrak{S}_{q}, q>0$ consists of all compact operators $A$, for which

$$
\|A\|_{q}:=\left[\sum_{k=1}^{\infty} s_{k}(A)^{q}\right]^{\frac{1}{q}}<\infty .
$$

If $q \geq 1$, then the above functional defines a norm. If $0<q<1$, then it is a quasinorm. There is nevertheless a convenient analogue of the triangle inequality, which is called the q-triangle inequality:

$$
\left\|A_{1}+A_{2}\right\|_{q}^{q} \leq\left\|A_{1}\right\|_{q}^{q}+\left\|A_{2}\right\|_{q}^{q}, \quad A_{1}, A_{2} \in \mathfrak{S}_{q}, \quad 0<q \leq 1
$$

see [Rot67] and also [BS87]. Thus $\|A\|_{q}$ is sometimes called a $q$-norm. Note also the Hölder inequality

$$
\left\|A_{1} A_{2}\right\|_{q} \leq\left\|A_{1}\right\|_{q_{1}} \cdot\left\|A_{2}\right\|_{q_{2}}, \quad q^{-1}=q_{1}^{-1}+q_{2}^{-1}, \quad 0<q_{1}, q_{2} \leq \infty .
$$

Further on we need some $\mathfrak{S}_{q}$-estimates for functions of self-adjoint operators that were established in [Sob]. As indicated in the Introduction, we are interested in functions that lose smoothness at finitely many points. Without loss of generality, 
for almost all estimates we may assume that $f$ has only one non-smoothness point. Below $\chi_{R}$ denotes the indicator function of the interval $(-R, R), R>0$. We impose the following condition.

Condition 2.1. For some integer $n \geq 1$ the function $f \in \mathrm{C}^{n}\left(\mathbb{R} \backslash\left\{t_{0}\right\}\right) \cap C(\mathbb{R})$ satisfies the bound

$$
|f|_{n}=\max _{0 \leq k \leq n} \sup _{t \neq t_{0}}\left|f^{(k)}(t)\right|\left|t-t_{0}\right|^{-\gamma+k}<\infty
$$

with some $\gamma>0$, and is supported on the interval $\left[t_{0}-R, t_{0}+R\right]$ with some $R>0$.

A function $f$ satisfying (2.2) with $n=1$ is Hölder-continuous:

$$
\left|f\left(t_{1}\right)-f\left(t_{2}\right)\right| \leq 2|f|_{1}\left|t_{1}-t_{2}\right|^{\varkappa}, \varkappa=\min \{\gamma, 1\}
$$

for all $t_{1}, t_{2} \in \mathbb{R}$. In what follows, all the bounds involving functions from Condition 2.1, are uniform in $t_{0}$, and contain explicit dependence on the quantity $(2.2)$, and on the radius $R$.

Now we can quote one abstract result following from [Sob, Theorem 2.10].

Proposition 2.2. Suppose that $f$ satisfies Condition 2.1 with some $\gamma>0, n \geq 2$ and some $t_{0} \in \mathbb{R}, R \in(0, \infty)$. Let $q$ be a number such that $(n-\sigma)^{-1}<q \leq 1$ with some number $\sigma \in(0,1], \sigma<\gamma$. Let $A$ be a bounded self-adjoint operator and let $P$ be an orthogonal projection such that $P A(I-P) \in \mathfrak{S}_{\sigma q}$. Then

$$
\|f(P A P) P-P f(A)\|_{q} \lesssim|f|_{n} R^{\gamma-\sigma}\|P A(I-P)\|_{\sigma q}^{\sigma},
$$

with an implicit constant independent of the operators $A, P$, the function $f$, and the parameters $R, t_{0}$.

Later in the proofs we apply this proposition to the operator (1.2).

We also need a version of Proposition 2.2 for smooth functions $f$, see [Sob, Corollary 2.11].

Proposition 2.3. Suppose that $g \in \mathrm{C}_{0}^{n}(-r, r)$, with some $r>0$ and $n \geq 2$. Assume $q \in(0,1]$ and $\sigma \in(0,1]$ are such that $(n-\sigma)^{-1}<q \leq 1$. Let the operator $A$ and orthogonal projection $P$ be as in Proposition 2.2. Then

$$
\|g(P A P) P-P g(A)\|_{q} \lesssim\|g\|_{C^{n}}\|P A(I-P)\|_{\sigma q}^{\sigma},
$$

with an implicit constant independent of the operator $A$, projection $P$ and the function $g$. 
2.2 The domains and regions. We always assume that $d \geq 2$. We say that $\Lambda$ is a basic Lipschitz (resp. basic $C^{m}, m=1,2, \ldots$ ) domain, if there is a Lipschitz (resp. $\mathrm{C}^{m}$ ) function $\Phi=\Phi(\hat{\mathbf{x}}), \hat{\mathbf{x}} \in \mathbb{R}^{d-1}$, such that with a suitable choice of Cartesian coordinates $\mathbf{x}=\left(\hat{\mathbf{x}}, x_{d}\right)$, the domain $\Lambda$ is represented as

$$
\Lambda=\left\{\mathbf{x} \in \mathbb{R}^{d}: x_{d}>\Phi(\hat{\mathbf{x}})\right\} .
$$

We use the notation $\Lambda=\Gamma(\Phi)$. The function $\Phi$ is assumed to be globally Lipschitz, i.e. the constant

$$
M_{\Phi}=\sup _{\hat{\mathbf{x}} \neq \hat{\mathbf{y}}} \frac{|\Phi(\hat{\mathbf{x}})-\Phi(\hat{\mathbf{y}})|}{|\hat{\mathbf{x}}-\hat{\mathbf{y}}|},
$$

is finite. Throughout the paper, all estimates involving basic Lipschitz domains $\Lambda=$ $\Gamma(\Phi)$, are uniform in the number $M_{\Phi}$.

A domain (i.e. connected open set) is said to be Lipschitz (resp. $\mathrm{C}^{m}$ ) if locally it coincides with some basic Lipschitz (resp. $C^{m}$ ) domain. We call $\Lambda$ a Lipschitz (resp. $\mathrm{C}^{m}$ ) region if $\Lambda$ is a union of finitely many Lipschitz (resp. $\mathrm{C}^{m}$ ) domains such that their closures are pair-wise disjoint.

A basic Lipschitz domain $\Lambda=\Gamma(\Phi)$ is said to be piece-wise $\mathrm{C}^{m}$ with some $m=1,2, \ldots$, if the function $\Phi$ is $C^{m}$-smooth away from a collection of finitely many $(d-2)$-dimensional Lipschitz surfaces $L_{1}, L_{2}, \cdots \subset \mathbb{R}^{d-1}$. By $(\partial \Lambda)_{\mathrm{s}} \subset \partial \Lambda$ we denote the set of points where the $C^{m}$-smoothness of the surface $\partial \Lambda$ may break down. A piece-wise $C^{m}$ region $\Lambda$ and the set $(\partial \Lambda)_{\mathrm{s}}$ for it are defined in the obvious way. An expanded version of these definitions can be found in [Sob14,Sob15], and here we omit the details.

The minimal assumptions on the sets featuring in this paper are laid out in the following condition.

Condition 2.4. The set $\Lambda \subset \mathbb{R}^{d}, d \geq 2$, is a Lipschitz region, and either $\Lambda$ or $\mathbb{R}^{d} \backslash \Lambda$ is bounded.

Some results, including the main asymptotic formulas in Theorem 2.7, require extra smoothness of $\Lambda$. Note that if $\Lambda$ is a Lipschitz (or $C^{m}$ ) region, then so is the interior of $\mathbb{R}^{d} \backslash \Lambda$.

2.3 The main result. We study the operator $D_{\alpha}\left(a_{T}, \Lambda ; f\right)$, see $(1.2)$ for the definition, with the symbol $a_{T}$ approximating the indicator function of an open set $\Omega \subset \mathbb{R}^{d}$. To state the precise definition of $a_{T}$ denote

$$
\rho(\boldsymbol{\xi})=\operatorname{dist}(\boldsymbol{\xi}, \partial \Omega), \tilde{\rho}(\boldsymbol{\xi})=\min \{\rho(\boldsymbol{\xi}), 1\},\langle t\rangle=\sqrt{1+t^{2}}, t \in \mathbb{R} .
$$

Condition 2.5. Let $\Omega \subset \mathbb{R}^{d}, d \geq 2$, satisfy Condition 2.4. The symbol $a_{T}=a_{T, \Omega} \in$ $\mathrm{C}^{\infty}\left(\mathbb{R}^{d}\right)$, depending on the set $\Omega$ and parameter $T \in\left(0, T_{0}\right]$, is a function satisfying the properties

$$
\left|a_{T}(\boldsymbol{\xi})-\chi_{\Omega}(\boldsymbol{\xi})\right| \lesssim\left\langle\rho(\boldsymbol{\xi}) T^{-1}\right\rangle^{-\beta}, \quad \beta>d
$$


and

$$
\left|\nabla^{m} a_{T}(\boldsymbol{\xi})\right| \lesssim(T+\tilde{\rho}(\boldsymbol{\xi}))^{-m}\left\langle\rho(\boldsymbol{\xi}) T^{-1}\right\rangle^{-\beta}, m=1,2, \ldots
$$

The implicit constants are independent of $T$, but may depend on $T_{0}$ and region $\Omega$.

Although $a_{T, \Omega}$ depends on two parameters, i.e. the number $T \in\left(0, T_{0}\right]$ and the region $\Omega \subset \mathbb{R}^{d}$, we usually omit the dependence on $\Omega$, since $\Omega$ is fixed.

The main results of this paper are contained in the next two theorems.

Theorem 2.6. Let $d \geq 2$. Suppose that $\Lambda \subset \mathbb{R}^{d}$ and $\Omega \subset \mathbb{R}^{d}$ satisfy Condition 2.4. Let $a_{T}=a_{T, \Omega} \in \mathrm{C}^{\infty}\left(\mathbb{R}^{d}\right)$ be a real-valued symbol depending on the parameter $T: 0<T \lesssim 1$, and satisfying Condition 2.5. Suppose also that $\alpha \gtrsim 1$.

Suppose that $f$ satisfies Condition 2.1 with $n=2$ and some $\gamma>0$. If $\beta>$ $\max \left\{d, d \gamma^{-1}\right\}$, then for any $\sigma \in\left(d \beta^{-1}, \min \{\gamma, 1\}\right)$, we have

$$
\left\|D_{\alpha}\left(a_{T}, \Lambda ; f\right)\right\|_{1} \lesssim R^{\gamma-\sigma} \alpha^{d-1} \log \left(\min \left\{\alpha, \frac{1}{T}\right\}+1\right)|f|_{2},
$$

with an implicit constant independent of $T, R, t_{0}, \alpha$ and the function $f$.

For the main asymptotic result we need some more notation. For any two Lipschitz regions $\Lambda, \Omega$ and a continuous function $b=b(\mathbf{x}, \boldsymbol{\xi})$ define the quantity

$$
\mathfrak{V}_{1}(b)=\mathfrak{V}_{1}(b ; \partial \Lambda, \partial \Omega)=\frac{1}{(2 \pi)^{d+1}} \int_{\partial \Lambda} \int_{\partial \Omega} b(\mathbf{x}, \boldsymbol{\xi})\left|\mathbf{n}_{\boldsymbol{\xi}} \cdot \mathbf{n}_{\mathbf{x}}\right| d S_{\boldsymbol{\xi}} d S_{\mathbf{x}}
$$

where $\mathbf{n}_{\mathbf{x}}, \mathbf{n}_{\boldsymbol{\xi}}$ are the exterior unit normals to the surfaces $\partial \Lambda$ and $\partial \Omega$ at the points $\mathbf{x}$ and $\boldsymbol{\xi}$ respectively. For a Hölder-continuous function $f: \mathbb{C} \mapsto \mathbb{C}$, define the integral

$$
U(f)=\int_{0}^{1} \frac{f(1-t)-(1-t) f(1)-t f(0)}{t(1-t)} d t .
$$

Now we can describe the asymptotics of $D_{\alpha}\left(a_{T}, \Lambda ; f\right)$.

Theorem 2.7. Let $d \geq 2$. Suppose that $\Lambda \subset \mathbb{R}^{d}$ and $\Omega \subset \mathbb{R}^{d}$ satisfy Condition 2.4. Suppose also that $\Lambda$ is piece-wise $C^{1}$, and $\Omega$ is piece-wise $C^{3}$.

Let $X=\left\{z_{1}, z_{2}, \ldots, z_{N}\right\} \subset \mathbb{R}, N<\infty$, be a collection of points on the real line. Suppose that $f \in C^{2}(\mathbb{R} \backslash X)$ is a function such that in a neighbourhood of each point $z \in X$ it satisfies the bound

$$
\left|f^{(k)}(t)\right| \leq C_{k}|t-z|^{\gamma-k}, k=0,1,2,
$$

with some $\gamma>0$.

Suppose that $a_{T}=a_{T, \Omega} \in \mathrm{C}^{\infty}\left(\mathbb{R}^{d}\right), 0<T \lesssim 1$, is a real-valued symbol as in Theorem 2.6. 
Then

$$
\lim _{\substack{T \rightarrow 0 \\ \alpha T \gtrsim 1}} \frac{1}{\alpha^{d-1} \log \frac{1}{T}} \operatorname{tr} D_{\alpha}\left(a_{T}, \Lambda ; f\right)=U(f) \mathfrak{V}_{1}(1 ; \partial \Lambda, \partial \Omega),
$$

and

$$
\lim _{\substack{\alpha \rightarrow \infty \\ \alpha T \lesssim 1}} \frac{1}{\alpha^{d-1} \log \alpha} \operatorname{tr} D_{\alpha}\left(a_{T}, \Lambda ; f\right)=U(f) \mathfrak{V}_{1}(1 ; \partial \Lambda, \partial \Omega) .
$$

REMARK 2.8. Note that in Theorems 2.6 and 2.7 either one or both regions $\Lambda$ or $\Omega$ are allowed to be unbounded, as long as the complements $\Omega^{c}$ and/or $\Lambda^{c}$ are bounded. Nevertheless, it suffices to prove Theorems 2.6 and 2.7 for a bounded $\Omega$ only. Indeed, suppose that $\Omega$ is a Lipschitz region such that $\Omega^{c}$ is bounded. Denote

$$
b_{T}=b_{T, \Omega^{c}}=1-a_{T, \Omega}, \quad g(t)=f(-t+1) .
$$

The symbol $b_{T}$ satisfies Condition 2.5 with $\Omega^{c}$ instead of $\Omega$. The function $g$ satisfies Condition 2.1 with $1-t_{0}$ instead of $t_{0}$ (for Theorem 2.6), or it satisfies the bound (2.13) for all $z \in \tilde{X}$ with $\tilde{X}=1-X$ (for Theorem 2.7). Moreover,

$$
f\left(W_{\alpha}\left(a_{T} ; \Lambda\right)\right) \chi_{\Lambda}=g\left(W_{\alpha}\left(b_{T} ; \Lambda\right)\right) \chi_{\Lambda}, f\left(\mathrm{Op}_{\alpha}\left(a_{T}\right)\right)=g\left(\mathrm{Op}_{\alpha}\left(b_{T}\right)\right),
$$

so that $D_{\alpha}\left(a_{T, \Omega}, \Lambda ; f\right)=D_{\alpha}\left(b_{T, \Omega^{c}}, \Lambda ; g\right)$. Thus Theorems 2.6 and 2.7 for the symbol $a_{T}$ and function $f$ follow from themselves for the symbol $b_{T}$ and function $g$.

This observation has no bearing on many intermediate estimates as our argument usually goes through equally well for bounded or unbounded $\Omega$. Nevertheless, it will be useful to us since some of the results that we borrow from the literature, have been formally proved for bounded $\Omega$ only.

REMARK 2.9. If the regions $\Lambda$ and $\Omega$ are bounded, and $f(0)=0$, then the second operator on the right-hand side of (1.2) is trace class, and its trace is easily found from the formula (see (1.3))

$$
\operatorname{tr} W_{\alpha}\left(f \circ a_{T} ; \Lambda\right)=\frac{\alpha^{d}}{(2 \pi)^{d}} \int_{\Lambda} \int_{\mathbb{R}^{d}} f\left(a_{T}(\boldsymbol{\xi})\right) d \boldsymbol{\xi} d \mathbf{x}=: \alpha^{d} \mathfrak{W}_{0}\left(a_{T} \chi_{\Lambda} ; f\right),
$$

and thus the formulas (2.14) and (2.15) give two-term asymptotics for $\operatorname{tr} f\left(W_{\alpha}\right)$. For instance, (2.14) can be rewritten as

$$
\begin{aligned}
\operatorname{tr} f\left(W_{\alpha}\left(a_{T} ; \Lambda\right)\right)= & \alpha^{d} \mathfrak{W}_{0}\left(a_{T} \chi_{\Lambda} ; f\right) \\
& +\alpha^{d-1} \log \frac{1}{T} U(f) \mathfrak{V}_{1}(1 ; \partial \Lambda, \partial \Omega) \\
& +o\left(\alpha^{d-1} \log \frac{1}{T}\right), \alpha T \gtrsim 1, T \rightarrow 0 .
\end{aligned}
$$

In this paper we discuss two possible realizations of the symbol $a_{T}$. The first example is given in the next subsection. 
2.4 Anti-Wick quantization. Let $\Omega$ be a Lipschitz region. One way to approximate $\chi_{\Omega}$ by a smooth function is to use the following standard procedure. Let $\zeta \in \mathrm{C}_{0}^{\infty}\left(\mathbb{R}^{d}\right)$ be a non-negative function with a support in the unit ball $B(\mathbf{0}, 1)$, such that $\|\zeta\|_{L^{1}}=1$. Define

$$
a_{T}(\boldsymbol{\xi})=\int_{\Omega} \zeta_{T}(\boldsymbol{\xi}-\boldsymbol{\eta}) d \boldsymbol{\eta}, \zeta_{T}(\boldsymbol{\xi})=\frac{1}{T^{d}} \zeta\left(\frac{\boldsymbol{\xi}}{T}\right) .
$$

It is straightforward that $a_{T}(\boldsymbol{\xi})-\chi_{\Omega}(\boldsymbol{\xi})=0$ for all $\boldsymbol{\xi}$ with the property $\rho(\boldsymbol{\xi})>T$, and $\left|\nabla^{m} a_{T}(\boldsymbol{\xi})\right| \lesssim T^{-m}, m=1,2, \ldots$, if $\rho(\boldsymbol{\xi}) \leq T$. Thus $a_{T}$ satisfies (2.8) and $(2.9)$ for any $\beta>0$, and hence Theorems 2.6 and 2.7 hold.

Clearly, the smoothing function $\zeta$ does not need to have a compact support. One can pick, for instance $\zeta(\boldsymbol{\xi})=c_{d} \exp \left(-|\boldsymbol{\xi}|^{2}\right)$ with the appropriate normalizing coefficient $c_{d}$. In this case the conditions (2.8) and (2.9) are also readily verified. With this choice of the smoothing $\zeta$, the symbol resembles the definition of the antiWick quantization of a pseudo-differential operator, see e.g. [Shu01, Ch. 4, §24]. Recall that if the anti-Wick symbol is given by $b(\mathbf{x}, \boldsymbol{\xi})$, then the "quasi-classical" Weyl symbol $b^{\mathrm{W}}$ of the same operator is found via the formula

$$
b_{T}^{\mathrm{W}}(\mathbf{x}, \boldsymbol{\xi})=\frac{1}{\left(\pi T^{2}\right)^{d}} \int \exp \left(-\frac{|\mathbf{x}-\mathbf{y}|^{2}}{T^{2}}-\frac{|\boldsymbol{\xi}-\boldsymbol{\eta}|^{2}}{T^{2}}\right) b(\mathbf{y}, \boldsymbol{\eta}) d \mathbf{y} d \boldsymbol{\eta} .
$$

Since (2.17) does not contain any dependence on the spatial variable, the symbol $a_{T}$ in fact is the Weyl symbol associated with the anti-Wick symbol $\chi_{\Omega}(\boldsymbol{\xi})$. At the same time, the indicator function $\chi_{\Lambda}$ in the definition (1.1) is not smoothed-out. Thus the operator $W_{\alpha}\left(a_{T} ; \Lambda\right)$ can be loosely described as a "partial" anti-Wick quantization. Therefore it seems appropriate to compare our results, e.g. formula (2.16), with the known asymptotic formulas for anti-Wick operators. These operators were introduced (see [Ber71]) and subsequently extensively studied in the case $T=$ $\alpha^{-1 / 2}$. We do not intend to discuss these studies in detail, and cite only the latest asymptotic result in this area, found in [Old15]. This paper was concerned with potentially discontinuous anti-Wick symbols of the form $b(\mathbf{x}, \boldsymbol{\xi})=\chi_{M}(\mathbf{x}, \boldsymbol{\xi}) \tilde{b}(\mathbf{x}, \boldsymbol{\xi})$ where $M \subset \mathbb{R}^{2 d}$ is a bounded smooth domain, and $\tilde{b}$ is a smooth symbol. For the sake of precision one should say that the paper [Old15] considered generalized antiWick-operators, i.e. those with arbitrary decreasing smoothing functions $\zeta$, but we do not elaborate on this point here. The main result of [Old15] states that for smooth functions $f$, such that $f(0)=0$, one has

$$
\operatorname{tr} f\left(\mathrm{Op}_{\alpha}\left(b_{T}^{W}\right)\right)=\alpha^{d} \mathfrak{W}_{0}\left(b_{T}^{W} ; f\right)+O\left(\alpha^{d-1}\right) \text { with } T=\frac{1}{\sqrt{\alpha}}, \quad \text { as } \quad \alpha \rightarrow \infty
$$

This formula, in contrast to (2.16), does not have a log-term. 


\section{The Fermi symbol: entanglement entropy and local entropy}

This section is focused on our second example of the symbol $a_{T}$. As mentioned earlier, the asymptotical problems studied in this paper are partly motivated by the study of entanglement entropy of free fermions both for positive and zero temperature $T$. In particular, for the positive temperature $T>0$ the relevant symbol $a_{T}$ is the Fermi symbol (1.7) with temperature $T>0$ and chemical potential $\mu>0$. The parameter $\mu$ is always assumed fixed. The assumptions on the function $h=h(\boldsymbol{\xi})$ are as follows:

Condition 3.1. (1) The function $h \in C^{\infty}\left(\mathbb{R}^{d}\right)$ is real-valued, and for sufficiently large $\boldsymbol{\xi}$ we have

$$
h(\boldsymbol{\xi}) \gtrsim|\boldsymbol{\xi}|^{\beta_{1}},
$$

with some constant $\beta_{1}>0$. Moreover,

$$
\left|\nabla^{n} h(\boldsymbol{\xi})\right| \lesssim(1+|\boldsymbol{\xi}|)^{\beta_{2}}, n=1,2, \ldots, \quad \forall \boldsymbol{\xi} \in \mathbb{R}^{d},
$$

with some $\beta_{2} \geq 0$.

(2) On the set $S=S_{\mu}=\left\{\boldsymbol{\xi} \in \mathbb{R}^{d}: h(\boldsymbol{\xi})=\mu\right\}$ the condition

$$
\nabla h(\boldsymbol{\xi}) \neq 0, \forall \boldsymbol{\xi} \in S .
$$

is satisfied.

(3) The set $\Omega_{\mu}=\left\{\boldsymbol{\xi} \in \mathbb{R}^{d}: h(\boldsymbol{\xi})<\mu\right\}$ has finitely many connected components.

Because of (3.1) the set $\Omega=\Omega_{\mu}$ is bounded, i.e. $\Omega \subset B\left(\mathbf{0}, R_{0}\right)$ with some $R_{0}>0$. Furthermore, due to the condition (3.3), the set $S_{\mu}$ is a $C^{\infty}$-surface (called the Fermi surface), and we have the bounds:

$$
|h(\boldsymbol{\xi})-\mu| \asymp \rho(\boldsymbol{\xi}), \quad \forall \boldsymbol{\xi} \in B\left(\mathbf{0}, R_{0}\right) .
$$

By virtue of (3.1) we also have the lower bound

$$
h(\boldsymbol{\xi})-\mu \gtrsim|\boldsymbol{\xi}|^{\beta_{1}},|\boldsymbol{\xi}| \geq R_{0} .
$$

Note the straightforward bound:

$$
\left|a_{T, \mu}(\boldsymbol{\xi})-\chi_{\Omega}(\boldsymbol{\xi})\right| \leq \exp \left(-\frac{|h(\boldsymbol{\xi})-\mu|}{T}\right), \boldsymbol{\xi} \in \mathbb{R}^{d} .
$$

This allows us to extend the definition (1.7) to $T=0$ :

$$
a_{0, \mu}(\boldsymbol{\xi})=\lim _{T \rightarrow 0} a_{T, \mu}(\boldsymbol{\xi})=\chi_{\Omega}(\boldsymbol{\xi}), \text { a.e. } \boldsymbol{\xi} \in \mathbb{R}^{d} .
$$

As pointed out in [LSS16b, Section 8], as a consequence of (3.1), (3.2) and (3.4), we also have

$$
\begin{aligned}
\left|\nabla^{n} a_{T, \mu}(\boldsymbol{\xi})\right| & \lesssim(T+\rho(\boldsymbol{\xi}))^{-n} \exp \left(-c_{1} \frac{\rho(\boldsymbol{\xi})}{T}\right), n=1,2, \ldots, \forall|\boldsymbol{\xi}| \leq R_{0}, \\
\left|\nabla^{n} a_{T, \mu}(\boldsymbol{\xi})\right| & \lesssim \exp \left(-c_{1} \frac{|\boldsymbol{\xi}|^{\beta_{1}}}{T}\right), n=1,2, \ldots, \forall|\boldsymbol{\xi}| \geq R_{0},
\end{aligned}
$$


with some positive constant $c_{1}$. Thus the symbol (1.7) satisfies the bounds (2.8) and (2.9) for any $\beta>0$. Consequently, the trace $\operatorname{tr} D_{\alpha}\left(a_{T, \mu}, \Lambda ; f\right)$ satisfies $(2.14)$ and (2.15). In order to study the entropy we use these asymptotic formulas with the $\gamma$-Rényi entropy function $\eta_{\gamma}: \mathbb{R} \mapsto[0, \infty)$ defined for all $\gamma>0$ as follows. If $\gamma \neq 1$, then

$$
\eta_{\gamma}(t):= \begin{cases}\frac{1}{1-\gamma} \log \left[t^{\gamma}+(1-t)^{\gamma}\right] & \text { for } t \in(0,1), \\ 0 & \text { for } t \notin(0,1)\end{cases}
$$

and for $\gamma=1$ (the von Neumann case) it is defined as the limit

$$
\eta_{1}(t):=\lim _{\gamma \rightarrow 1} \eta_{\gamma}(t)= \begin{cases}-t \log (t)-(1-t) \log (1-t) & \text { for } t \in(0,1), \\ 0 & \text { for } t \notin(0,1) .\end{cases}
$$

For $\gamma \neq 1$ the function $\eta_{\gamma}$ satisfies condition (2.13) with $\gamma$ replaced with $\varkappa=$ $\min \{\gamma, 1\}$, and with $X=\{0,1\}$. The function $\eta_{1}$ satisfies (2.13) with an arbitrary $\gamma \in(0,1)$, and the same set $X$.

We begin with reminding definitions of the entropies in the form given in [LSS16b, Section 10]. If $\Lambda \subset \mathbb{R}^{d}$ is bounded, then the local (thermal) $\gamma$-Rényi entropy of the equilibrium state at temperature $T \geq 0$ and chemical potential $\mu \in \mathbb{R}$ is defined as

$$
\mathrm{S}_{\gamma}(T, \mu ; \Lambda):=\operatorname{tr}\left[\eta_{\gamma}\left(W_{1}\left(a_{T, \mu} ; \Lambda\right)\right)\right] .
$$

For arbitrary $\Lambda \subset \mathbb{R}^{d}$ we define the $\gamma$-Rényi entanglement entropy (EE) with respect to the bipartition $\mathbb{R}^{d}=\Lambda \cup\left(\mathbb{R}^{d} \backslash \Lambda\right)$, as

$$
\mathrm{H}_{\gamma}(T, \mu ; \Lambda):=\operatorname{tr} D_{1}\left(a_{T, \mu}, \Lambda ; \eta_{\gamma}\right)+\operatorname{tr} D_{1}\left(a_{T, \mu}, \mathbb{R}^{d} \backslash \Lambda ; \eta_{\gamma}\right)
$$

These entropies were studied in [LSS] (for $T=0$ ) and [LSS16b] (for $T>0$ ). In particular, in [LSS16b] it was shown that for any $T>0$ the EE is finite, if $\Lambda$ satisfies Condition 2.4.

We are interested in the behaviour of the above quantities when $T \rightarrow 0$ and $\Lambda$ is replaced with $\alpha \Lambda$, with a large scaling parameter $\alpha$. The next theorem establishes sharp bounds for the entropies (3.7) and (3.8).

Theorem 3.2. Let $d \geq 2$. Suppose that $\Lambda$ satisfies Condition 2.4. Suppose that $0<T \lesssim 1$ and $\alpha \gtrsim 1$. Then the $\gamma$-Rényi entanglement entropy satisfies

$$
\left|\mathrm{H}_{\gamma}(T, \mu ; \alpha \Lambda)\right| \lesssim \alpha^{d-1} \log \left(\min \left\{\alpha, T^{-1}\right\}+1\right) .
$$

If $\Lambda$ is bounded, then the local $\gamma$-Rényi entropy satisfies

$$
\left|\mathrm{S}_{\gamma}(T, \mu ; \alpha \Lambda)-\alpha^{d} s_{\gamma}(T, \mu)\right| \Lambda|| \lesssim \alpha^{d-1} \log \left(\min \left\{\alpha, T^{-1}\right\}+1\right),
$$

where

$$
s_{\gamma}(T, \mu):=\frac{1}{(2 \pi)^{d}} \int \eta_{\gamma}\left(a_{T, \mu}(\boldsymbol{\xi})\right) d \boldsymbol{\xi} .
$$

The constants in (3.9) and (3.10) are independent of $\alpha$ and $T$, but may depend on the function $h$, parameter $\mu$ and the region $\Lambda$. 
The coefficient $s_{\gamma}(T, \mu)$ is called the $\gamma$-Rényi entropy density (cf. [LSS]). For $\alpha T \gtrsim 1$ the bounds (3.9) and (3.10) were derived in [LSS16b].

Proof of Theorem 3.2. In view of additivity of the operator $D_{\alpha}(a, \Lambda ; f)$ in the functional parameter $f$, the bound (3.9) follows from Theorem 2.6.

In order to prove (3.10), we rewrite (3.7):

$$
\mathrm{S}_{\gamma}(T, \mu ; \alpha \Lambda)=\operatorname{tr}\left[\chi_{\Lambda} \eta_{\gamma}\left(\mathrm{Op}_{\alpha}\left(a_{T, \mu}\right)\right) \chi_{\Lambda}\right]+\operatorname{tr} D_{\alpha}\left(a_{T, \mu}, \Lambda ; \eta_{\gamma}\right)
$$

For the first trace we use the identity (1.3), and for the second one - the bound (3.9). This yields (3.10).

The next theorem establishes the asymptotic behaviour of the local entropy and of the EE.

Theorem 3.3. Suppose that $\Lambda \subset \mathbb{R}^{d}, d \geq 2$, satisfies Condition 2.4, and is piecewise $\mathrm{C}^{1}$. Then the EE satisfies

$$
\lim _{\substack{T \rightarrow 0 \\ \alpha T \gtrsim 1}} \frac{1}{\alpha^{d-1} \log \frac{1}{T}} \mathrm{H}_{\gamma}(T, \mu ; \alpha \Lambda)=2 \pi^{2} \frac{1+\gamma}{6 \gamma} \mathfrak{V}_{1}(1 ; \partial \Lambda, \partial \Omega),
$$

and

$$
\lim _{\substack{\alpha \rightarrow \infty \\ \alpha T \lesssim 1}} \frac{1}{\alpha^{d-1} \log \alpha} \mathrm{H}_{\gamma}(T, \mu ; \alpha \Lambda)=2 \pi^{2} \frac{1+\gamma}{6 \gamma} \mathfrak{V}_{1}(1 ; \partial \Lambda, \partial \Omega) .
$$

If the region $\Lambda$ is bounded, then the local entropy satisfies

$$
\lim _{\substack{T \rightarrow 0 \\ \alpha T \gtrsim 1}} \frac{1}{\alpha^{d-1} \log \frac{1}{T}}\left(S_{\gamma}(T, \mu ; \alpha \Lambda)-\alpha^{d} s_{\gamma}(T, \mu)|\Lambda|\right)=\pi^{2} \frac{1+\gamma}{6 \gamma} \mathfrak{V}_{1}(1 ; \partial \Lambda, \partial \Omega),
$$

and

$$
\lim _{\substack{\alpha \rightarrow \infty \\ \alpha T \lesssim 1}} \frac{1}{\alpha^{d-1} \log \alpha}\left(\mathrm{S}_{\gamma}(T, \mu ; \alpha \Lambda)-\alpha^{d} s_{\gamma}(T, \mu)|\Lambda|\right)=\pi^{2} \frac{1+\gamma}{6 \gamma} \mathfrak{V}_{1}(1 ; \partial \Lambda, \partial \Omega) .
$$

Proof. Formulas (3.11) and (3.12) follow from (2.14) and (2.15) respectively upon observing (cf. [LSS]) that

$$
U\left(\eta_{\gamma}\right)=\int_{0}^{1} \frac{\eta_{\gamma}(t)}{t(1-t)} d t=\pi^{2} \frac{1+\gamma}{6 \gamma}
$$

Formulas (3.13) and (3.14) also follow from (2.14) and (2.15), and from (1.3).

For $d=1$ and $\alpha T \gtrsim 1$ Theorem 3.3 was proved in [LSS16b]. We also re-iterate that the formulas above agree with the large-scale asymptotics of the entropies $\mathrm{H}_{\gamma}$ and $\mathrm{S}_{\gamma}$ for the zero temperature case, which were found in [LSS]. 


\section{Smooth functions of self-adjoint operators}

4.1 The Helffer-Sjöstrand formula. When studying functions of self-adjoint operators we rely on the Helffer-Sjöstrand formula (see [HS89]) which holds for arbitrary operator $X=X^{*}$ and arbitrary smooth function $f \in C_{0}^{n}(\mathbb{R}), n \geq 2$ :

$$
f(X)=\frac{1}{\pi} \iint z(x, y)(X-x-i y)^{-1} d x d y, z(x, y)=\frac{\partial}{\partial \bar{z}} \tilde{f}(x, y)
$$

where $\tilde{f}=\tilde{f}(x, y)$ is a quasi-analytic extension of the function $f$, see [Dav95, Ch. 2]. A quasi-analytic extension of $f \in C^{n}(\mathbb{R})$ is a $C^{1}\left(\mathbb{R}^{2}\right)$-function $\tilde{f}$, such that $f(x)=$ $\tilde{f}(x, 0)$ and $|\mathcal{Z}(x, y)| \leq C|y|$. For the sake of brevity we use the representation (4.1) for compactly supported functions only, so that that the integral (4.1) is normconvergent.

Let us describe a convenient quasi-analytic extension of a function $f \in \mathrm{C}_{0}^{n}(\mathbb{R})$. For an arbitrary $r>0$ introduce the function

$$
U(x, y)=\left\{\begin{array}{l}
1,|y|<\langle x\rangle, \quad\langle x\rangle=\sqrt{x^{2}+1} . \\
0,|y| \geq\langle x\rangle,
\end{array}\right.
$$

The next proposition can be found in [Dav95, Ch. 2].

Proposition 4.1. Let $f \in \mathrm{C}^{n}(\mathbb{R}), n \geq 2$. Then the function $f$ has a quasi-analytic extension $\tilde{f}=\tilde{f}(\cdot, \cdot) \in C^{1}\left(\mathbb{R}^{2}\right)$ such that $\tilde{f}(x, y)=0$ if $|y|>\langle x\rangle$. Moreover, the derivative

$$
z(x, y)=\frac{\partial}{\partial \bar{z}} \tilde{f}(x, y)
$$

satisfies the bound

$$
|Z(x, y)| \lesssim F(x)|y|^{n-1} U(x, y)
$$

where

$$
F(x)=\sum_{l=0}^{n}\left|f^{(l)}(x)\right|\langle x\rangle^{-n+l} .
$$

The constant in (4.3) does not depend on $f$.

Denote

$$
N_{n}(f)=\int F(x)\langle x\rangle^{n-2} d x
$$


4.2 Quasi-commutators. Let $\mathfrak{H}$ be a Hilbert space. Let $A, B: \mathfrak{H} \mapsto \mathfrak{H}$ be some bounded self-adjoint operators, and let $J: \mathfrak{H} \mapsto \mathfrak{H}$ be a bounded operator. Here we make some elementary observations about the "quasi-commutator"

$$
f(A) J-J f(B)
$$

with a smooth function $f$. We are interested in estimates in the normed ideal $\mathfrak{S}$ of compact operators with the norm $\|\cdot\| \mathfrak{s}$.

Theorem 4.2. Let $A, B$ be two self-adjoint bounded operators, and let $J$ be a bounded operator. Suppose that $f \in \mathrm{C}_{0}^{n}(\mathbb{R})$ with $n \geq 3$. Let $\mathfrak{S}$ be a normed ideal of compact operators acting on $\mathfrak{H}$. Then

$$
\|f(A) J-J f(B)\|_{\mathfrak{S}} \lesssim N_{n}(f)\|A J-J B\|_{\mathfrak{S}} .
$$

The constant in (4.5) does not depend on $f$ or $A, B, J$.

Proof. Let us consider the function $f(t)=r_{z}(t)=(t-z)^{-1}$ with $\operatorname{Im} z \neq 0$, and prove that

$$
\left\|r_{z}(A) J-J r_{z}(B)\right\|_{\mathfrak{S}} \leq \frac{1}{|\operatorname{Im} z|^{2}}\|A J-J B\|_{\mathfrak{S}}
$$

By the resolvent identity

$$
r_{z}(A) J-J r_{z}(B)=-r_{z}(A)(A J-J B) r_{z}(B),
$$

we have

$$
\left\|r_{z}(A) J-J r_{z}(B)\right\|_{\mathfrak{S}} \leq \frac{1}{|\operatorname{Im} z|^{2}}\|A J-J B\|_{\mathfrak{S}},
$$

whence (4.6).

By formula (4.1) and Proposition 4.1, we have

$$
\begin{aligned}
\|f(A) J-J f(B)\|_{\mathfrak{S}} \\
\quad \lesssim \iint_{|y|<\langle x\rangle} F(x)|y|^{n-1}\left\|r_{x+i y}(A) J-J r_{x+i y}(B)\right\|_{\mathfrak{S}} d x d y \\
\quad \lesssim\|A J-J B\|_{\mathfrak{S}} \iint_{|y|<\langle x\rangle} F(x)|y|^{n-3} d x d y,
\end{aligned}
$$

where we have used (4.6). The right-hand side is clearly estimated by $N_{n}(f) \| A J-$ $J B \|_{\mathfrak{S}}$, as required. 
From now on, unless otherwise stated we always assume that $f \in \mathrm{C}_{0}^{n}(\mathbb{R})$ with some $n \geq 3$. We apply the above simple result to the operators of the form

$$
\mathcal{D}\left(A_{1}, P_{1} ; f\right) J-J \mathcal{D}\left(A_{2}, P_{2} ; f\right),
$$

involving two pairs of self-adjoint bounded operators $A_{1}, A_{2}$ and $P_{1}, P_{2}$, where

$$
\mathcal{D}(A, P ; f)=P f(P A P) P-P f(A) P .
$$

We do not consider the most general case, but concentrate on a very special one, which is used later in the proof of the main theorem.

As before, the constants in the estimates below depend neither on the function $f$ nor operators involved.

Corollary 4.3. Let $A_{1}, A_{2}$ be bounded self-adjoint operators, and let $P_{1}, P_{2}$ be bounded self-adjoint operators such that $\left\|P_{1}\right\|,\left\|P_{2}\right\| \leq 1$. Let $J$ be a bounded operator. Suppose that

$$
\left[P_{1}, J\right]=\left[P_{2}, J\right]=0
$$

and

$$
\left(P_{1}-P_{2}\right) J=0
$$

Then

$$
\begin{aligned}
& \left\|\mathcal{D}\left(A_{1}, P_{1} ; f\right) J-J \mathcal{D}\left(A_{2}, P_{2} ; f\right)\right\|_{\mathfrak{S}} \\
& \quad \lesssim N_{n}(f)\left[\left\|\left(A_{1}-A_{2}\right) J\right\|_{\mathfrak{S}}+\left\|\left[A_{2}, J\right]\right\|_{\mathfrak{S}}\right]
\end{aligned}
$$

and

$$
\begin{aligned}
& \left\|J \mathcal{D}\left(A_{1}, P_{1} ; f\right)-J \mathcal{D}\left(A_{2}, P_{2} ; f\right)\right\|_{\mathfrak{S}} \\
& \quad \lesssim N_{n}(f)\left[\left\|\left(A_{1}-A_{2}\right) J\right\|_{\mathfrak{S}}+\left\|\left[A_{1}, J\right]\right\|_{\mathfrak{S}}+\left\|\left[A_{2}, J\right]\right\|_{\mathfrak{S}}\right] .
\end{aligned}
$$

Proof. Observe first that (4.11) follows from (4.10) due to the following equality:

$$
\begin{aligned}
& J \mathcal{D}\left(A_{1}, P_{1} ; f\right)-J \mathcal{D}\left(A_{2}, P_{2} ; f\right) \\
& \quad=\left[J, \mathcal{D}\left(A_{1}, P_{1} ; f\right)\right]+\mathcal{D}\left(A_{1}, P_{1} ; f\right) J-J \mathcal{D}\left(A_{2}, P_{2} ; f\right) .
\end{aligned}
$$

Proof of (4.10). By (4.8),

$$
\left\|\left[P_{1} A_{2} P_{2}, J\right]\right\|_{\mathfrak{S}} \leq\left\|\left[A_{2}, J\right]\right\|_{\mathfrak{S}} .
$$

Also, by (4.5),

$$
\left\|\left[f\left(P_{2} A_{2} P_{2}\right), J\right]\right\|_{\mathfrak{S}} \lesssim N_{n}(f)\left\|\left[A_{2}, J\right]\right\|_{\mathfrak{S}} .
$$


Thus, in view of (4.8) and (4.9),

$$
\begin{array}{rl}
P_{1} & f\left(P_{1} A_{1} P_{1}\right) P_{1} J-J P_{2} f\left(P_{2} A_{2} P_{2}\right) P_{2} \\
= & P_{1}\left(f\left(P_{1} A_{1} P_{1}\right) J-J f\left(P_{2} A_{2} P_{2}\right)\right) P_{1} \\
& \quad+P_{1}\left[f\left(P_{2} A_{2} P_{2}\right), J\right] P_{2}-P_{1}\left[f\left(P_{2} A_{2} P_{2}\right), J\right] P_{1} .
\end{array}
$$

By (4.5) and (4.8), (4.9), the first term on the right-hand side does not exceed

$$
\begin{aligned}
& N_{n}(f)\left\|P_{1} A_{1} P_{1} J-J P_{1} A_{2} P_{2}\right\|_{\mathfrak{S}} \\
& \quad \leq N_{n}(f)\left(\left\|P_{1} A_{1} P_{1} J-P_{1} A_{2} P_{1} J\right\|_{\mathfrak{S}}+\left\|\left[P_{1} A_{2} P_{2}, J\right]\right\|_{\mathfrak{S}}\right) \\
& \quad \leq N_{n}(f)\left(\left\|\left(A_{1}-A_{2}\right) J\right\|_{\mathfrak{S}}+\left\|\left[A_{2}, J\right]\right\|_{\mathfrak{S}}\right) .
\end{aligned}
$$

The second and the third terms are bounded by $N_{n}(f)\left\|\left[A_{2}, J\right]\right\|_{\mathfrak{S}}$. This leads to (4.10).

Corollary 4.4. Let $A$ be a bounded self-adjoin operator, and let $P$ be an orthogonal projection. Let $J$ be an operator such that $[P, J]=0$. Then

$$
\|J \mathcal{D}(A, P ; f)\|_{\mathfrak{S}}+\|\mathcal{D}(A, P ; f) J\|_{\mathfrak{S}} \lesssim N_{n}(f)\left(\|J P A(I-P)\|_{\mathfrak{S}}+\|[A, J]\|_{\mathfrak{S}}\right) .
$$

The constant in (4.12) is independent of $A, P, J$ or $f$.

Proof. It suffices to prove (4.12) for the operator $\mathcal{D}(A, P ; f) J$ only. Rewrite it:

$$
\begin{aligned}
\mathcal{D}(A, P ; f) J & =P f(P A P) P J-P f(A) J P \\
& =P f(P A P) P J-P J f(A) P-P[f(A), J] P \\
& =P(f(P A P) P J-P J f(A)) P-P[f(A), J] P .
\end{aligned}
$$

By (4.5), the $\mathfrak{S}$-norm of the second term is bounded by $N_{n}(f) \|\left[A, J \|_{\mathfrak{S}}\right.$. By $(4.5)$, the $\mathfrak{S}$-norm of the first term is bounded by

$$
N_{n}(f)\|P A P J-P J A\|_{\mathfrak{S}} \leq N_{n}(f)\left(\|J P A(I-P)\|_{\mathfrak{S}}+\|[A, J]\|_{\mathfrak{S}}\right) .
$$

This completes the proof of (4.12).

4.3 Elementary estimates for pseudo-differential operators. Now we apply Corollaries 4.3 and 4.4 to pseudo-differential operators. As above we assume that $f \in \mathrm{C}_{0}^{n}(\mathbb{R})$ with some $n \geq 3$. The quantity $N_{n}(f)$ is defined in (4.4).

Below we always assume that $\varphi \in \mathrm{C}^{\infty}\left(\mathbb{R}^{d}\right)$ is a bounded function. Often we also assume that for some sets $\Lambda$ and $\Pi$,

$$
\operatorname{supp} \varphi \cap \Lambda=\operatorname{supp} \varphi \cap \Pi \text {. }
$$


Lemma 4.5. Let $b, \tilde{b}$ be some real-valued symbols, and let $\Lambda$ and $\Pi$ be some sets. Then

$$
\left\|\varphi D_{\alpha}(b, \Lambda ; f)\right\|_{1} \lesssim N_{n}(f)\left(\left\|\chi_{\Lambda} \varphi \mathrm{Op}_{\alpha}(b)\left(I-\chi_{\Lambda}\right)\right\|_{1}+\left\|\left[\mathrm{Op}_{\alpha}(b), \varphi\right]\right\|_{1}\right) .
$$

Suppose that (4.13) is satisfied. Then

$$
\begin{aligned}
& \left\|\varphi D_{\alpha}(b, \Lambda ; f)-\varphi D_{\alpha}(\tilde{b}, \Pi ; f)\right\|_{1} \\
& \quad \lesssim N_{n}(f)\left(\left\|\left[\varphi, \mathrm{Op}_{\alpha}(b)\right]\right\|_{1}+\left\|\varphi \mathrm{Op}_{\alpha}(b-\tilde{b})\right\|_{1}+\left\|\mathrm{Op}_{\alpha}(b-\tilde{b}) \varphi\right\|_{1}\right) .
\end{aligned}
$$

If, in addition, $\Pi=\mathbb{R}^{d}$, then

$$
\left\|\varphi D_{\alpha}(b, \Lambda ; f)\right\|_{1} \lesssim N_{n}(f)\left\|\left[\varphi, \mathrm{Op}_{\alpha}(b)\right]\right\|_{1} .
$$

The constants in the above bounds do not depend on the function $f, \varphi$, sets $\Lambda, \Pi$ or symbols $b, \tilde{b}$.

Proof. The bound (4.14) follows from Corollary 4.4 with $P=\chi_{\Lambda}, A=\mathrm{Op}_{\alpha}(b)$, $J=\varphi$.

The bound (4.16) follows from (4.15) used with $b=\tilde{b}$, since $D_{\alpha}\left(b, \mathbb{R}^{d} ; f\right)=0$.

Proof of (4.15). Use Corollary 4.3 with

$$
A_{1}=\mathrm{Op}_{\alpha}(b), \quad A_{2}=\mathrm{Op}_{\alpha}(\tilde{b}), \quad P_{1}=\chi_{\Lambda}, \quad P_{2}=\chi_{\Pi}, \quad J=\varphi .
$$

The condition (4.8) is trivially satified. By (4.13), the condition (4.9) is also satisfied. Thus (4.15) follows from (4.11).

\section{Estimates for Wiener-Hopf operators}

In this section we collect some Schatten-von Neumann bounds for Wiener-Hopf operators with symbols satisfying some general conditions. Our main objective is to ensure the explicit dependence of the bounds on the symbols.

5.1 Some basic bounds. To control the scaling properties of functions we introduce the following norms:

$$
\mathbf{N}^{(n)}(\eta ; \tau)=\max _{0 \leq k \leq n} \sup _{\boldsymbol{\xi}} \tau^{k}\left|\nabla_{\boldsymbol{\xi}}^{k} \eta(\boldsymbol{\xi})\right|, \quad n=1,2, \ldots
$$

First we give some bounds in Schatten-von Neumann classes $\mathfrak{S}_{q}, q \in(0,1]$, established in [Sob14], but adjusted for our purposes in the current paper.

Unless specified otherwise, below each of the sets $\Lambda, \Omega \subset \mathbb{R}^{d}$ is a Lipschitz region. If $\Lambda$ (or $\Omega$ ) is a basic Lipschitz domain, i. e. $\Lambda=\Gamma(\Phi)$ with a globally Lipschitz function $\Phi$, then the constants in the estimates obtained below are uniform in $\Lambda$ in the sense that they depend only on the constant $M$ in the bound $M_{\Phi} \leq M$.

Below we often use a test-function $\varphi \in \mathrm{C}_{0}^{\infty}\left(\mathbb{R}^{d}\right)$ such that

$$
\text { support of the function } \varphi \text { is contained in } B(\mathbf{z}, \ell) \text {, }
$$


with some constant $\ell>0$ and some $\mathbf{z} \in \mathbb{R}^{d}$. The bounds below are uniform in $\mathbf{z}$, since by translation one can always assume that $\mathbf{z}=\mathbf{0}$. The constants in all estimates are independent of the parameters $\alpha, \ell$ and $\tau$.

Lemma 5.1. Suppose that $\varphi$ satisfies (5.2), and that the support of $\eta \in \mathrm{C}_{0}^{\infty}\left(\mathbb{R}^{d}\right)$ is contained in a ball of radius $\tau>0$. Let $q \in(0,1]$ and

$$
r=r_{q}=\left[(d+1) q^{-1}\right]+1 .
$$

(1) Let $\Lambda$ and $\Omega$ be Lipschitz regions. If $\alpha \ell \tau \gtrsim 1$, then

$$
\begin{aligned}
& \left\|\left[\varphi \mathrm{Op}_{\alpha}(\eta), \chi_{\Lambda}\right]\right\|_{q}+\left\|\left[\varphi \mathrm{Op}_{\alpha}(\eta), \mathrm{Op}_{\alpha}\left(\chi_{\Omega}\right)\right]\right\|_{q} \\
& \quad \lesssim(\alpha \ell \tau)^{\frac{d-1}{q}} \mathrm{~N}^{(r)}(\varphi ; \ell) \mathrm{N}^{(r)}(\eta ; \tau) .
\end{aligned}
$$

(2) Let $\Lambda$ be a Lipschitz region, and let $\Omega$ satisfy Condition 2.4. Suppose that $\alpha \ell \gtrsim 1$. Then

$$
\left\|\chi_{\Lambda} \chi_{B(\mathbf{z}, \ell)} \mathrm{Op}_{\alpha}\left(\chi_{\Omega}\right)\left(I-\chi_{\Lambda}\right)\right\|_{q} \lesssim\left[(\alpha \ell)^{d-1} \log (\alpha \ell+1)\right]^{\frac{1}{q}} .
$$

(3) Let $\Lambda$ and $\Omega$ satisfy Condition 2.4. If $\alpha \ell \gtrsim 1$, then

$$
\left\|\left[\varphi, \mathrm{Op}_{\alpha}\left(\chi_{\Omega}\right)\right]\right\|_{q} \lesssim(\alpha \ell)^{\frac{d-1}{q}} \mathrm{~N}^{(r)}(\varphi ; \ell) .
$$

If $\alpha \tau \gtrsim 1$, then

$$
\left\|\left[\mathrm{Op}_{\alpha}(\eta), \chi_{\Lambda}\right]\right\|_{q} \lesssim(\alpha \tau)^{\frac{d-1}{q}} \mathrm{~N}^{(r)}(\eta ; \tau) .
$$

If $\Lambda$ (or $\Omega$ ) is basic Lipschitz, then the relevant bounds are uniform in $\Lambda$ (or $\Omega$ ).

Proof. The bounds (5.4) follow from [Sob14, Theorem 4.2 and Corollary 4.4].

For bounded $\Omega$ the bound (5.5) is easily deduced from [Sob14, Theorem 4.6 and Corollary 4.7]. We omit the details. If $\Omega^{c}=\mathbb{R}^{d} \backslash \Omega$ is bounded, then

$$
\chi_{\Lambda} \varphi \mathrm{Op}_{\alpha}\left(\chi_{\Omega}\right)\left(I-\chi_{\Lambda}\right)=-\chi_{\Lambda} \varphi \mathrm{Op}_{\alpha}\left(\chi_{\Omega^{c}}\right)\left(I-\chi_{\Lambda}\right),
$$

and we can use [Sob14] again.

For bounded $\Lambda$ and $\Omega$ the bounds (5.6) and (5.7) follow from (5.4) by using a suitable partition of unity, or one can use the appropriate result from [Sob14, Corollary 4.4]. In the case of bounded complements $\Lambda^{c}$ and $\Omega^{c}$ we use the obvious identities

$$
\left.\left[\varphi, \mathrm{Op}_{\alpha}\left(\chi_{\Omega}\right)\right]=-\left[\varphi, \mathrm{Op}_{\alpha}\left(\chi_{\Omega^{c}}\right)\right], \quad\left[\mathrm{Op}_{\alpha}(\eta), \chi_{\Lambda}\right]=-\left[\mathrm{Op}_{\alpha}(\eta), \chi_{\Lambda^{c}}\right)\right],
$$

and [Sob14, Corollary 4.4] again.

Lemma 5.2. Let $a=a(\boldsymbol{\xi})$ be a symbol, and let $\ell, \alpha>0$ be some numbers. Then for any $m \geq d+1$ and any $s \geq s_{0}>1$,

$$
\left\|\chi_{B(\mathbf{z}, \ell)} \operatorname{Op}_{\alpha}(a)\left(1-\chi_{B(\mathbf{z}, s \ell)}\right)\right\|_{1} \lesssim((s-1) \alpha \ell)^{d-m}\left\|\nabla^{m} a\right\|_{L^{1}} .
$$

The implicit constant does not depend on $\ell, \alpha, \varphi$ and $a$, but may depend on $s_{0}$. 
Proof. Without loss of generality we may assume that $\mathbf{z}=\mathbf{0}$. The operator in (5.8) is unitarily equivalent to

$$
\chi_{B(\mathbf{0}, 1)} \mathrm{Op}_{1}(\tilde{a})\left(I-\chi_{B(\mathbf{0}, s)}\right), \tilde{a}(\boldsymbol{\xi})=a\left(\boldsymbol{\xi}(\alpha \ell)^{-1}\right) .
$$

Since the sets $B(\mathbf{0}, 1)$ and $\mathbb{R}^{d} \backslash B(\mathbf{0}, s)$ are separated by a positive distance $s-1$, it follows from [Sob14, Theorem 2.6] that

$$
\left\|\chi_{B(\mathbf{0}, 1)} \mathrm{Op}_{1}(\tilde{a})\left(I-\chi_{B(\mathbf{0}, s)}\right)\right\|_{1} \lesssim(s-1)^{d-m}\left\|\nabla^{m} \tilde{a}\right\|_{\mathrm{L}^{1}},
$$

for any $m \geq d+1$. The $\mathrm{L}^{1}$-norm on the right-hand side coincides with $(\alpha \ell)^{d-m} \| \nabla^{m}$ $a \|_{L^{1}}$, which leads to (5.8).

5.2 Bounds for more general operators. For methodological purposes it is also necessary to introduce more general pseudo-differential operators. For a function $p=p(\mathbf{x}, \mathbf{y}, \boldsymbol{\xi})$, which we call amplitude, define the operator

$$
\left(\mathrm{Op}_{\alpha}^{\mathrm{a}}(p) u\right)(\mathbf{x})=\frac{\alpha^{d}}{(2 \pi)^{\frac{d}{2}}} \iint e^{i \alpha \boldsymbol{\xi} \cdot(\mathbf{x}-\mathbf{y})} p(\mathbf{x}, \mathbf{y}, \boldsymbol{\xi}) u(\mathbf{y}) d \mathbf{y} d \boldsymbol{\xi}, u \in \mathrm{S}\left(\mathbb{R}^{d}\right) .
$$

We need a very simple-looking bound for the trace norm of $\mathrm{Op}_{\alpha}^{\mathrm{a}}(p)$ which we borrow from [Sob14, Theorem 2.5]:

LEMMA 5.3.

$$
\left\|\mathrm{Op}_{1}^{\mathrm{a}}(p)\right\|_{1} \lesssim \sum_{n, l=0}^{d+1} \iiint\left|\nabla_{\mathbf{x}}^{n} \nabla_{\mathbf{y}}^{l} p(\mathbf{x}, \mathbf{y}, \boldsymbol{\xi})\right| d \mathbf{x} d \mathbf{y} d \boldsymbol{\xi}
$$

and

$$
\left\|\mathrm{Op}_{\alpha}^{\mathrm{a}}(p)\right\|_{1} \lesssim \alpha^{d} \sum_{n, l=0}^{d+1} \iiint\left|\nabla_{\mathbf{x}}^{n} \nabla_{\mathbf{y}}^{l} p(\mathbf{x}, \mathbf{y}, \boldsymbol{\xi})\right| d \mathbf{x} d \mathbf{y} d \boldsymbol{\xi}
$$

for any $\alpha>0$. The implicit constants in (5.9) and (5.10) do not depend on $\alpha$ or amplitude $p$.

Proof. The bound (5.9) is a direct consequence of [Sob14, Theorem 2.5]. The bound (5.10) follows from (5.9) by rescaling $\boldsymbol{\xi} \mapsto \boldsymbol{\xi} \alpha^{-1}$.

The above estimates are convenient for us because they do not contain any derivatives w.r.t. $\boldsymbol{\xi}$.

Lemma 5.4. Let $a=a(\boldsymbol{\xi})$, and let $\varphi=\varphi(\mathbf{x})$ satisfy (5.2). Then for any $\alpha>0$ and $\ell>0$, we have

$$
\left\|\left[\mathrm{Op}_{\alpha}(a), \varphi\right]\right\|_{1} \lesssim(\alpha \ell)^{d-1} \mathrm{~N}^{(d+2)}(\varphi ; \ell)\left[\|\nabla a\|_{\mathrm{L}^{1}}+(\alpha \ell)^{1-m}\left\|\nabla^{m} a\right\|_{\mathrm{L}^{1}}\right],
$$

with an arbitrary $m \geq d+1$. The implicit constant in (5.11) does not depend on $\alpha, \ell, \varphi$ or $a$. 
Proof. Without loss of generality assume that $\mathbf{z}=0$ and $\ell=1$. Let $\tilde{\varphi} \in \mathrm{C}_{0}^{\infty}\left(\mathbb{R}^{d}\right)$ be a function such that $\tilde{\varphi}(\mathbf{x})=1$ for $|\mathbf{x}|<2$. Denote

$$
\tilde{p}(\mathbf{x}, \mathbf{y}, \boldsymbol{\xi})=a(\boldsymbol{\xi}) \tilde{\varphi}(\mathbf{x})(\varphi(\mathbf{x})-\varphi(\mathbf{y})) \tilde{\varphi}(\mathbf{y}) .
$$

Then by (5.8),

$$
\left\|\left[\varphi, \mathrm{Op}_{\alpha}(a)\right]-\mathrm{Op}_{\alpha}^{\mathrm{a}}(\tilde{p})\right\|_{1} \lesssim \alpha^{d-m} \mathrm{~N}^{(d+1)}(\varphi ; 1)\left\|\nabla^{m} a\right\|_{\mathrm{L}^{1}},
$$

for any $m \geq d+1$. Further proof we conduct for $d=1$, although we do not replace $d$ by its value in the estimates below. The case of arbitrary $d$ is done in a similar way with obvious modifications. Integrate by parts in $\xi$, so that

$$
\mathrm{Op}_{\alpha}^{\mathrm{a}}(\tilde{p})=-\frac{1}{i \alpha} \mathrm{Op}_{\alpha}^{\mathrm{a}}(p), p(x, y, \xi)=\frac{\partial_{\xi} \tilde{p}(x, y, \xi)}{x-y} .
$$

Rewrite

$$
p(x, y, \xi)=a^{\prime}(\xi) \tilde{\varphi}(x) \tilde{\varphi}(y) \int_{0}^{1} \varphi^{\prime}(x+t(y-x)) d t .
$$

Thus by (5.10),

$$
\left\|\mathrm{Op}_{\alpha}^{\mathrm{a}}(\tilde{p})\right\|_{1} \lesssim \alpha^{d-1} \mathrm{~N}^{(d+1)}(\nabla \varphi ; 1)\|\nabla a\|_{\mathrm{L}^{1}}
$$

where the implicit constant depends on $\tilde{\varphi}$. Together with (5.12) this gives (5.11).

5.3 Multi-scale symbols. The bounds above are very convenient as they contain easily computable quantities, such as integral norms of symbol's derivatives. We also need other types of bounds where the dependence on the symbol $a$ is less explicit, but still sufficient for our needs. Following [LSS16b], we achieve this by placing ourselves in the context of multi-scale symbols.

Let $v=v(\boldsymbol{\xi})$ and $\tau=\tau(\boldsymbol{\xi})$ be some continuous, positive functions on $\mathbb{R}^{d}$. Consider a symbol $a \in \mathrm{C}^{\infty}\left(\mathbb{R}^{d}\right)$ satisfying the bounds

$$
\left|\nabla_{\boldsymbol{\xi}}^{k} a(\boldsymbol{\xi})\right| \lesssim \tau(\boldsymbol{\xi})^{-k} v(\boldsymbol{\xi}), k=0,1,2, \ldots, \quad \boldsymbol{\xi} \in \mathbb{R}^{d} .
$$

It is natural to call $\tau$ a scale (function) and $v$ the amplitude (function). We always assume that $\|v\|_{\mathrm{L}^{\infty}} \leq 1$ and

$$
\tau_{\mathrm{inf}}:=\inf _{\boldsymbol{\xi} \in \mathbb{R}^{d}} \tau(\boldsymbol{\xi})>0
$$

Introduce the notation

$$
V_{\sigma, \omega}(v, \tau):=\int \frac{v(\boldsymbol{\xi})^{\sigma}}{\tau(\boldsymbol{\xi})^{\omega}} d \boldsymbol{\xi}, \sigma>0, \omega \in \mathbb{R} .
$$

Apart from the continuity we need some extra conditions on the scale and the amplitude. First we assume that $\tau$ is globally Lipschitz, i.e., for some $\nu \in(0,1)$,

$$
|\tau(\boldsymbol{\xi})-\tau(\boldsymbol{\eta})| \leq \nu|\boldsymbol{\xi}-\boldsymbol{\eta}|, \quad \boldsymbol{\xi}, \boldsymbol{\eta} \in \mathbb{R}^{d}
$$


with some $\nu>0$. By adjusting the implicit constants in (5.13) one may choose for $\nu$ an arbitrary positive value. We assume that this value can be picked in such a way that the amplitude $v$ satisfies the relation

$$
\frac{v(\boldsymbol{\eta})}{v(\boldsymbol{\xi})} \asymp 1, \boldsymbol{\eta} \in B(\boldsymbol{\xi}, \tau(\boldsymbol{\xi})) .
$$

In the next result we establish some bounds that depend explicitly on the functional parameters $v$ and $\tau$.

The following result follows from [LSS16b, Lemma 3.4 and Theorem 3.5].

Proposition 5.5. Let a be a symbol satisfying (5.13) with some scaling function $\tau$ and some amplitude $v(\boldsymbol{\xi})$ for which (5.16), (5.14) and (5.17) hold. Suppose that $\Lambda$ is a Lipschitz region, and that $\alpha \ell \tau_{\text {inf }} \gtrsim 1$. Then

$$
\left\|\chi_{\Lambda} \chi_{B(\mathbf{z}, \ell)} \mathrm{Op}_{\alpha}(a)\left(I-\chi_{\Lambda}\right)\right\|_{q}^{q} \lesssim(\alpha \ell)^{d-1} V_{q, 1}(v, \tau) .
$$

If $\Lambda$ is basic Lipschitz, then this bound is uniform in $\Lambda$.

Suppose in addition that

- $\Lambda$ satisfies Condition 2.4,

- the function $f$ satisfies Condition 2.1 with some $\gamma>0, R>0$ and $n=2$, and that $\alpha \tau_{\mathrm{inf}} \gtrsim 1$.

Then for any $\sigma<\min \{1, \gamma\}$, we have

$$
\left\|D_{\alpha}(a, \Lambda ; f)\right\|_{1} \lesssim \alpha^{d-1}|f|_{2} R^{\gamma-\sigma} V_{\sigma, 1}(v, \tau) .
$$

The implicit constants in (5.18) and (5.19) do not depend on $\alpha, f$ and $R$, but depend on the region $\Lambda$, on the implicit constants in (5.13), (5.17), and on the parameter $\nu$.

\section{Bounds involving the symbol $a_{T}$ : Proof of Theorem 2.6}

6.1 Elementary bounds for the symbol $\boldsymbol{a}_{\boldsymbol{T}}$. Let $a_{T}=a_{T, \Omega}$ be a symbol satisfying Condition 2.5, with a bounded $\Omega$. In order to derive some integral bounds for $a_{T}$ we need to obtain estimates for the function $\rho(\boldsymbol{\xi}$ ) (see $(2.7)$ ) in terms of the Lipschitz functions responsible for the local representation of $\partial \Omega$.

Since the region $\Omega$ is bounded and has finitely many connected components, we can cover the boundary $S=\partial \Omega$ with finitely many open balls $\left\{D_{j}\right\}$ of equal radii $r \leq 1$, centred at some $\boldsymbol{\xi}_{j} \in S$, such that in each of the balls $D_{j}$ the boundary $S$, with an appropriate choice of coordinates, is the graph of a Lipschitz function $\Psi_{j}$ on $\mathbb{R}^{d-1}$ :

$$
S \cap D_{j}=\left\{\boldsymbol{\xi} \in \mathbb{R}^{d}: \xi_{d}=\Psi_{j}(\hat{\boldsymbol{\xi}})\right\} \cap D_{j} .
$$

We may also assume that

$$
\Omega \cap D_{j}=\left\{\boldsymbol{\xi} \in \mathbb{R}^{d}: \xi_{d}>\Psi_{j}(\hat{\boldsymbol{\xi}})\right\} \cap D_{j} .
$$


Let $\tilde{D}$ be an open subset of $\mathbb{R}^{d} \backslash S$, such that

$$
\mathbb{R}^{d}=\left(\cup_{j} D_{j}\right) \cup \tilde{D}
$$

It is clear that one can choose the balls $D_{j}$ so that

$$
\left\{\begin{array}{l}
\rho(\boldsymbol{\xi}) \asymp\left|\xi_{d}-\Psi_{j}(\hat{\boldsymbol{\xi}})\right|, \quad \boldsymbol{\xi} \in D_{j}, \\
\rho(\boldsymbol{\xi}) \asymp\langle\boldsymbol{\xi}\rangle, \boldsymbol{\xi} \in \tilde{D} .
\end{array}\right.
$$

It is natural to view $a_{T}$ as a multi-scale symbol (see Subsect. 5.3 for the definition). Indeed, the bounds (2.8) and (2.9) imply that

$$
\left|\nabla^{m} a_{T}(\boldsymbol{\xi})\right| \lesssim(T+\tilde{\rho}(\boldsymbol{\xi}))^{-m}\langle\boldsymbol{\xi}\rangle^{-\beta}, \quad m=0,1,2, \ldots,
$$

so $a_{T}$ satisfies (5.13) with

$$
v(\boldsymbol{\xi})=\langle\boldsymbol{\xi}\rangle^{-\beta}, \quad \tau(\boldsymbol{\xi})=\frac{1}{2}(\tilde{\rho}(\boldsymbol{\xi})+T) .
$$

Since $|\nabla \tilde{\rho}|=1$ a.e., the thus defined scale $\tau$ satisfies (5.16) with $\nu=1 / 2$. Furthermore, the function $v$ satisfies (5.17). Note also that $\tau_{\text {inf }} \asymp T$.

Lemma 6.1. Let $a_{T}=a_{T, \Omega}$ be as in Condition 2.5, and let $0<T \lesssim 1$. Then for any $\delta>d \beta^{-1}$

$$
\left\|\left|a_{T}-\chi_{\Omega}\right|^{\delta}\right\|_{L^{1}} \lesssim T
$$

and for any $m \geq 1$,

$$
\left\|\nabla^{m} a_{T}\right\|_{L^{1}} \lesssim T^{-m+1}
$$

Furthermore, let $v$ and $\tau$ be as defined in (6.6). Then for any $\sigma>d \beta^{-1}$,

$$
V_{\sigma, 1}(v, \tau) \lesssim \log \left(\frac{1}{T}+1\right)
$$

and

$$
V_{\sigma, \omega}(v, \tau) \lesssim T^{-\omega+1}, \forall \omega>1
$$

The implicit constants in (6.7), (6.8), (6.9) and (6.10) depend only on the constants in (2.5).

Proof. Proof of (6.7). We estimate separately the integrals over domains $D_{j}$ and $\tilde{D}$. By (2.8) and (6.4), the integral over $\tilde{D}$ does not exceed

$$
\int_{\tilde{D}}\left\langle|\boldsymbol{\xi}| T^{-1}\right\rangle^{-\delta \beta} d \boldsymbol{\xi} \lesssim T^{d} \int_{0}^{\infty}\langle s\rangle^{-\delta \beta} s^{d-1} d s \lesssim T^{d} .
$$


In the same way, in view of (6.4), the integral over $D_{j}$ is bounded by

$$
\int_{D_{j}}\left\langle\left|\xi_{d}-\Psi_{j}(\hat{\boldsymbol{\xi}})\right| T^{-1}\right\rangle^{-\delta \beta} d \boldsymbol{\xi} \lesssim \int_{0}^{\infty}\left\langle s T^{-1}\right\rangle^{-\delta \beta} d s \lesssim T .
$$

The bound (6.8) is proved in a similar way: using (6.4) and (2.9) we conclude that

$$
\int_{\tilde{D}}\left|\nabla^{m} a_{T}(\boldsymbol{\xi})\right| d \boldsymbol{\xi} \lesssim \int\left\langle|\boldsymbol{\xi}| T^{-1}\right\rangle^{-\beta} d \boldsymbol{\xi} \lesssim T^{d}
$$

and

$$
\begin{aligned}
\int_{D_{j}}\left|\nabla^{m} a_{T}(\boldsymbol{\xi})\right| d \boldsymbol{\xi} & \lesssim \int_{D_{j}}\left(T+\left|\xi_{d}-\Psi_{j}(\hat{\boldsymbol{\xi}})\right|\right)^{-m}\left\langle\left|\xi_{d}-\Psi_{j}(\hat{\boldsymbol{\xi}})\right| T^{-1}\right\rangle^{-\beta} d \boldsymbol{\xi} \\
& \lesssim T^{1-m} \int_{0}^{\infty}(1+s)^{-m-\beta} d s
\end{aligned}
$$

as required.

Proof of (6.9) and (6.10). As above, we use the covering (6.3) and estimate the integrals over $D_{j}, \tilde{D}$ separately, so that

$$
\begin{aligned}
\int_{D_{j}}(T+\tilde{\rho}(\boldsymbol{\xi}))^{-\omega}\langle\boldsymbol{\xi}\rangle^{-\sigma \beta} d \boldsymbol{\xi} & \lesssim \int_{D_{j}}\left(T+\left|\xi_{d}-\Psi_{j}(\hat{\boldsymbol{\xi}})\right|\right)^{-\omega} d \boldsymbol{\xi} \\
& \lesssim \int_{0}^{1} \frac{1}{(T+s)^{\omega}} d s \lesssim\left\{\begin{array}{l}
\log \left(\frac{1}{T}+1\right), \omega=1, \\
T^{1-\omega}, \omega>1,
\end{array}\right.
\end{aligned}
$$

and

$$
\int_{\tilde{D}}(T+\tilde{\rho}(\boldsymbol{\xi}))^{-\omega}\langle\boldsymbol{\xi}\rangle^{-\sigma \beta} d \boldsymbol{\xi} \lesssim \int\langle\boldsymbol{\xi}\rangle^{-\sigma \beta} d \boldsymbol{\xi} \lesssim 1 .
$$

This proves (6.9) and (6.10).

6.2 Bounds for pseudo-differential operators with $\boldsymbol{a}_{T}$. Without delay we infer the following useful consequence of the above bounds.

LEMMA 6.2. Let $\Omega$ be a bounded Lipschitz region. Let $\alpha, \ell>0$ be some numbers and let $0<T \lesssim 1$. If $\varphi$ satisfies (5.2), then for arbitrary $m \geq d+1$, and any $s>1$,

$$
\left\|\chi_{B(\mathbf{z}, \ell)} \mathrm{Op}_{\alpha}\left(a_{T}\right)\left(I-\chi_{B(\mathbf{z}, s \ell)}\right)\right\|_{1} \lesssim(\alpha \ell)^{d-1}(\alpha \ell T)^{1-m}
$$

and

$$
\left\|\left[\varphi, \mathrm{Op}_{\alpha}\left(a_{T}\right)\right]\right\|_{1} \lesssim(\alpha \ell)^{d-1} \mathrm{~N}^{(d+2)}(\varphi ; \ell)\left(1+(\alpha \ell T)^{1-m}\right) .
$$

The implicit constants in (6.11) and (6.12) are independent of $\alpha, \ell, T$ or $\varphi$. 
Proof. The bound (6.11) follows from (5.8) and (6.8). The bound (6.12) follows from (5.11) and (6.8).

Let us use Proposition 5.5 for the symbol $a_{T}$.

Proposition 6.3. Suppose that $\Lambda$ is a Lipschitz region, and that $\Omega$ is a bounded Lipschitz region. If $\alpha \ell T \gtrsim 1$, then for any $q \in\left(d \beta^{-1}, 1\right]$ we have

$$
\left\|\chi_{\Lambda} \chi_{B(\mathbf{z}, \ell)} \operatorname{Op}_{\alpha}\left(a_{T}\right)\left(I-\chi_{\Lambda}\right)\right\|_{q}^{q} \lesssim(\alpha \ell)^{d-1} \log \left(\frac{1}{T}+1\right) .
$$

If $\Lambda$ is basic Lipschitz, then this bound is uniform in $\Lambda$. Suppose in addition that

- $\Lambda$ satisfies Condition 2.4,

- $f$ satisfies Condition 2.1 with some $\gamma>0, R>0$ and $n=2$, and that $\beta>\max \left\{d \gamma^{-1}, d\right\}$.

If $\alpha T \gtrsim 1$, then for any $\sigma \in\left(d \beta^{-1}, \gamma\right), \sigma<1$ :

$$
\left\|D_{\alpha}\left(a_{T}, \Lambda ; f\right)\right\|_{1} \lesssim \alpha^{d-1}|f|{ }_{2} R^{\gamma-\sigma} \log \left(\frac{1}{T}+1\right) .
$$

The implicit constants in (6.13) and (6.14) are independent of $\alpha, \ell, T$ and $f$.

Proof. Since $\tau_{\text {inf }} \asymp T$, we have $\alpha \ell \tau_{\text {inf }} \asymp \alpha \ell T \gtrsim 1$. So the bounds (5.18) and (6.9) lead to (6.13). Under the condition $\alpha \tau_{\text {inf }} \asymp \alpha T \gtrsim 1$ the bounds (5.19) and (6.9) lead to (6.14).

6.3 Lattice norm bounds for pseudo-differential operators. For a function $u: \mathbb{R}^{d} \mapsto \mathbb{C}$ denote

$$
\|u\|_{q}=\left[\sum_{\mathbf{n} \in \mathbb{Z}^{d}}\left(\int_{\mathbf{n}+[0,1)^{d}}|u(\mathbf{x})|^{2} d \mathbf{x}\right)^{\frac{q}{2}}\right]^{\frac{1}{q}} .
$$

If $q \geq 1$, this formula defines a norm, sometimes called a lattice norm, and if $q<1$, then-quasi-norm, called lattice quasi-norm. The following result is wellknown, see [BS77, Theorem 11.1], [BKS91, Section 5.8], and for $q \in[1,2)$ - [Sim05, Theorem 4.5].

Proposition 6.4. If $\|w\|_{q},\|b\|_{q}<\infty$ for some $q \in(0,2]$, then

$$
\left\|w \mathrm{Op}_{1}(b)\right\|_{q} \lesssim\|w\|_{q}\|b\|_{q} .
$$

Corollary 6.5. Let $\alpha, \ell>0$ and $0<T \lesssim 1$ be such that $\alpha \ell \gtrsim 1$ and $\alpha T \ell \lesssim 1$. Let $\Omega \subset \mathbb{R}^{d}$ and $a_{T}=a_{T, \Omega}$ be as in Condition 2.5. Then for any $q \in\left(d \beta^{-1}, 1\right]$

$$
\left\|\chi_{B(\mathbf{z}, \ell)} \operatorname{Op}_{\alpha}\left(a_{T}-\chi_{\Omega}\right)\right\|_{q}^{q} \lesssim(\alpha \ell)^{d-1},
$$

with an implicit constant independent of $\mathbf{z} \in \mathbb{R}^{d}$ or $\alpha, \ell, T$. 
Proof. Let $\left\{\phi_{j}\right\}, \tilde{\phi}$ be a partition of unity subordinate to the cover (6.3). Denote for brevity $a=a_{T}$. Estimate separately the operators

$$
Z_{j}=\chi_{B(\mathbf{z}, \ell)} \operatorname{Op}_{\alpha}\left(\phi_{j}\left(a-\chi_{\Omega}\right)\right) \text { and } \tilde{Z}=\chi_{B(\mathbf{z}, \ell)} \mathrm{Op}_{\alpha}\left(\tilde{\phi}\left(a-\chi_{\Omega}\right)\right)
$$

Let $\Psi=\Psi_{j} \in \operatorname{Lip}\left(\mathbb{R}^{d-1}\right)$ be a function describing the surface $S$ inside $D_{j}$, see (6.1). Recall that we always assume that $\|\nabla \Psi\|_{L^{\infty}} \lesssim 1$. Without loss of generality assume that $\mathbf{z}=0$. By rescaling, the operator $Z_{j}$ is unitarily equivalent to

$$
Z_{j}^{\prime}=\chi_{B(\mathbf{0}, 1)} \mathrm{Op}_{1}(b), b(\boldsymbol{\xi})=\left[a\left(\boldsymbol{\xi}(\alpha \ell)^{-1}\right)-\chi_{\Omega}\left(\boldsymbol{\xi}(\alpha \ell)^{-1}\right)\right] \phi_{j}\left(\boldsymbol{\xi}(\alpha \ell)^{-1}\right),
$$

By Proposition 6.4,

$$
\left\|\chi_{B(\mathbf{0}, 1)} \mathrm{Op}_{1}(b)\right\|_{q} \lesssim\left\|\chi_{B(\mathbf{0}, 1)}\right\|_{q}\|b\|_{q} .
$$

It is clear that $\| \chi_{B(\mathbf{0}, 1)} \boldsymbol{\|}_{q}<\infty$ and it is independent of any parameters. Let us estimate $\|b\|_{q}$. By (2.8),

$$
|b(\boldsymbol{\xi})| \lesssim\left\langle\frac{\left|\xi_{d}-\tilde{\Psi}(\hat{\boldsymbol{\xi}})\right|}{\alpha \ell T}\right\rangle^{-\beta} \chi_{B(\mathbf{0}, \alpha \ell)}(\boldsymbol{\xi}), \tilde{\Psi}(\boldsymbol{\xi})=\alpha \ell \Psi\left(\hat{\boldsymbol{\xi}}(\alpha \ell)^{-1}\right) .
$$

Define the sets

$$
\mathcal{O}_{s}=\left\{\boldsymbol{\xi} \in B(\mathbf{0}, \alpha \ell): s \leq\left|\xi_{d}-\tilde{\Psi}(\hat{\boldsymbol{\xi}})\right|<s+1\right\}, s=0,1, \ldots
$$

Since $\|\nabla \tilde{\Psi}\|_{L^{\infty}}=\|\nabla \Psi\|_{L^{\infty}} \lesssim 1$, the number

$$
\#\left\{\mathbf{n} \in \mathbb{Z}^{d}: \mathbf{n}+[0,1)^{d} \cap \mathcal{O}_{s} \neq \varnothing\right\}
$$

does not exceed $(\alpha \ell)^{d-1}$, uniformly in $s$. As a result,

$$
\left\|b \chi_{\odot_{s}}\right\|_{q} \lesssim\langle s\rangle^{-\beta}(\alpha \ell)^{\frac{d-1}{q}}
$$

where we have used the property $\alpha \ell T \lesssim 1$. Thus, by the $q$-triangle inequality, we have

$$
\|b\|_{q}^{q} \leq \sum_{s}\left\|b \chi_{\mathcal{O}_{s}}\right\|_{q}^{q} \lesssim(\alpha \ell)^{d-1} \sum_{s}\langle s\rangle^{-\beta q} \lesssim(\alpha \ell)^{d-1}
$$

Let us now consider the operator $\tilde{Z}=\chi_{B(\mathbf{0}, \ell)} \mathrm{Op}_{\alpha}(\tilde{b}), \tilde{b}=\tilde{\phi}\left(a-\chi_{\Omega}\right)$. By rescaling, the operator $\tilde{Z}$ is unitarily equivalent to

$$
\tilde{Z}^{\prime}=\chi_{B(\mathbf{0}, \alpha \ell)} \mathrm{Op}_{1}(\tilde{b}),
$$

By virtue of $(6.4), \rho(\boldsymbol{\xi}) \gtrsim|\boldsymbol{\xi}|, \boldsymbol{\xi} \in \tilde{D}$, and hence (2.8) implies that

$$
\|\tilde{b}\|_{q}^{q} \lesssim \sum_{\mathbf{n} \in \mathbb{Z}^{d}}\left\langle\mathbf{n} T^{-1}\right\rangle^{-\beta q} \lesssim \int_{0}^{\infty}\left\langle\frac{s}{T}\right\rangle^{-\beta q} s^{d-1} d s=T^{d} \int_{0}^{\infty}\langle s\rangle^{-\beta q} s^{d-1} d s \lesssim T^{d} .
$$


Furthermore, since $\alpha \ell \gtrsim 1$, we have

$$
\| \chi_{B(\mathbf{0}, \alpha \ell)} \boldsymbol{\|}_{q}^{q} \lesssim(\alpha \ell)^{d} .
$$

By Proposition 6.4,

$$
\|\tilde{Z}\|_{q}^{q} \lesssim(\alpha \ell)^{d} T^{d} \lesssim(\alpha \ell T)^{d} \lesssim 1
$$

Collecting the contributions from all $D_{j}$ 's and $\tilde{D}$, we get the bound (6.15), as claimed.

Corollary 6.6. Let $\varphi$ satisfy (5.2), and let $\Omega$ be a bounded Lipschitz region. Suppose that $\alpha \ell \gtrsim 1$. Then for any $0<T \lesssim 1$ we have

$$
\left\|\left[\varphi, \mathrm{Op}_{\alpha}\left(a_{T}\right)\right]\right\|_{1} \lesssim \mathrm{N}^{(d+2)}(\varphi ; \ell)(\alpha \ell)^{d-1} .
$$

Moreover, for any Lipschitz region $\Lambda$, and any $q \in\left(d \beta^{-1}, 1\right]$ we have

$$
\left\|\chi_{\Lambda} \chi_{B(\mathbf{z}, \ell)} \mathrm{Op}_{\alpha}\left(a_{T}\right)\left(I-\chi_{\Lambda}\right)\right\|_{q}^{q} \lesssim(\alpha \ell)^{d-1} \log \left(\min \left\{\alpha \ell, \frac{1}{T}\right\}+1\right) .
$$

The constants in (6.16) and (6.17) are independent of $\alpha, \ell, T$ or $\varphi$.

Proof. The bound (6.16) holds for $\alpha \ell T \gtrsim 1$, due to (6.12). For $\alpha \ell T \lesssim 1$ we use (6.15) and (5.6) to get

$$
\begin{aligned}
\left\|\left[\varphi, \mathrm{Op}_{\alpha}\left(a_{T}\right)\right]\right\|_{1} & \leq\left\|\left[\varphi, \mathrm{Op}_{\alpha}\left(\chi_{\Omega}\right)\right]\right\|_{1}+2\left\|\varphi \mathrm{Op}_{\alpha}\left(a_{T}-\chi_{\Omega}\right)\right\|_{1} \\
& \lesssim \mathrm{N}^{(d+2)}(\varphi ; \ell)(\alpha \ell)^{d-1}
\end{aligned}
$$

Thus (6.16) is proved.

Proof of (6.17). If $\alpha \ell T \gtrsim 1$, then (6.17) follows directly from (6.13). For $\alpha \ell T \lesssim 1$ estimate using the $q$-triangle inequality (2.1):

$$
\begin{aligned}
\left\|\chi_{\Lambda} \chi_{B(\mathbf{z}, \ell)} \mathrm{Op}_{\alpha}\left(a_{T}\right)\left(I-\chi_{\Lambda}\right)\right\|_{q}^{q} \leq & \left\|\chi_{B(\mathbf{z}, \ell)} \mathrm{Op}_{\alpha}\left(a_{T}-\chi_{\Omega}\right)\right\|_{q}^{q} \\
& +\left\|\chi_{\Lambda} \chi_{B(\mathbf{z}, \ell)} \mathrm{Op}_{\alpha}\left(\chi_{\Omega}\right)\left(I-\chi_{\Lambda}\right)\right\|_{q}^{q} .
\end{aligned}
$$

By (6.15) and (5.5), the right-hand side satisfies the required bound.

6.4 Proof of Theorem 2.6. Recall that by Remark 2.8 it suffices to prove Theorem 2.6 for a bounded $\Omega$. In this case the bound (2.10) for $\alpha T \gtrsim 1$ is already proved in Proposition 6.3.

Suppose that $\alpha T \lesssim 1$. It immediately follows from Proposition 2.2 with $P=$ $\chi_{\Lambda}, A=\mathrm{Op}_{\alpha}\left(a_{T}\right)$, that

$$
\left\|D\left(a_{T}, \Lambda ; f\right)\right\|_{1} \lesssim|f|_{2} R^{\gamma-q}\left\|\chi_{\Lambda} \mathrm{Op}_{\alpha}\left(a_{T}\right)\left(I-\chi_{\Lambda}\right)\right\|_{q}^{q},
$$

for any $q<\min \{1, \gamma\}$. Let $B(\mathbf{0}, R)$ be a ball such that either $\Lambda \subset B(\mathbf{0}, R)$ or $\mathbb{R}^{d} \backslash \Lambda \subset B(\mathbf{0}, R)$. Thus the $\mathfrak{S}_{q}$-norm on the right-hand side is estimated either by

$$
\left\|\chi_{B(\mathbf{0}, R)} \chi_{\Lambda} \mathrm{Op}_{\alpha}\left(a_{T}\right)\left(I-\chi_{\Lambda}\right)\right\|_{q}^{q}
$$


or by

$$
\|\left[\chi_{\Lambda} \mathrm{Op}_{\alpha}\left(a_{T}\right) \chi_{B(\mathbf{0}, R)}\left(I-\chi_{\Lambda}\right) \|_{q}^{q}\right.
$$

For $q \in\left(d \beta^{-1}, \gamma\right), q \leq 1$ both (quasi-)norms are bounded as in (6.17). This leads to the proclaimed bound (2.10).

REMARK 6.7. In the proof of the main Theorem 2.7 we also need a version of the bound (2.10) for smooth functions $f$. Suppose that $g \in \mathrm{C}_{0}^{2}(-r, r), r>0$. Then,arguing as in the proof above but using Proposition 2.3 instead of Proposition 2.2 , we obtain the bound

$$
\left\|D\left(a_{T}, \Lambda ; g\right)\right\|_{1} \lesssim\|g\|_{C^{2}}\left\|\chi_{\Lambda} \mathrm{Op}_{\alpha}\left(a_{T}\right)\left(I-\chi_{\Lambda}\right)\right\|_{q}^{q}
$$

for any $q<1$, with an implicit constant independent of $g$, but dependent on the number $r$. As in the proof above, this bound in combination with (6.17) leads to the estimate

$$
\left\|D\left(a_{T}, \Lambda ; g\right)\right\|_{1} \lesssim\|g\|_{C^{2}} \alpha^{d-1} \log \left(\min \left\{\alpha, \frac{1}{T}\right\}+1\right)
$$

\section{Asymptotics for discontinuous symbols}

In the proof of the main theorem we use two types of asymptotics for $D_{\alpha}\left(\chi_{\Omega}, \Lambda ; g_{p}\right)$ for polynomials $g_{p}(t)=t^{p}, p=1,2, \ldots$, established in [Sob13] and [Sob15]. Recall that the integrals $\mathfrak{V}_{1}(b ; \partial \Lambda, \partial \Omega)$ and $U(f)$ are defined in (2.11) and (2.12) respectively.

In the case $\alpha T \lesssim 1$ we need the following fact, see [Sob15, Lemma 4.3].

Proposition 7.1. Let $\Lambda$ and $\Omega$ be regions in $\mathbb{R}^{d}$ satisfying Condition 2.4, and let $\Omega$ be bounded. Assume also that $\Omega$ is piece-wise $C^{3}$ and $\Lambda$ is piece-wise $C^{1}$. Then for any function $\varphi \in \mathrm{C}_{0}^{\infty}\left(\mathbb{R}^{d}\right)$, we have

$$
\lim _{\alpha \rightarrow \infty} \frac{1}{\alpha^{d-1} \log \alpha} \operatorname{tr}\left(\varphi D_{\alpha}\left(\chi_{\Omega}, \Lambda ; g_{p}\right)\right)=U\left(g_{p}\right) \mathfrak{V}_{1}(\varphi ; \partial \Lambda, \partial \Omega) .
$$

Under the condition $\alpha T \gtrsim 1$ we appeal to more subtle results from [Sob13]. These are not stated in [Sob13] exactly in the required form, hence we need to do some extra work. Let $\Lambda=\Gamma(\Phi)$ be a basic $C^{1}$-domain. We need to control the modulus of continuity of $\nabla \Phi$, hence we assume that

$$
\sup _{\hat{\mathbf{x}}, \hat{\mathbf{y}}:|\hat{\mathbf{x}}-\hat{\mathbf{y}}|<r}|\nabla \Phi(\hat{\mathbf{x}})-\nabla \Phi(\hat{\mathbf{y}})| \leq \varepsilon(r)
$$

for some non-negative function $\varepsilon$ such that $\varepsilon(r) \rightarrow 0$ as $r \rightarrow 0$. 
REMARK 7.2. The asymptotic formula stated in the next Proposition is uniform in the basic domain $\Lambda$ in the sense that the convergence is uniform in all functions $\Phi$ satisfying the bound $M_{\Phi} \leq M$ and (7.1) with some constant $M$ and some function $\varepsilon=\varepsilon(r)$. In particular, the domain $\Lambda$ is allowed to depend on the large parameter $\alpha$ as long as the bounds $M_{\Phi} \leq M$ and (7.1) hold with some $\alpha$-independent $M$ and $\varepsilon(r)$.

Proposition 7.3. Let $\Lambda$ be a basic $C^{1}$-domain, and let $\Omega$ be a bounded $\mathrm{C}^{3}$-region. Let $g_{p}(t)=t^{p}, t \in \mathbb{R}, p=1,2, \ldots$, and let $\varphi=\varphi(\mathbf{x}), \eta=\eta(\boldsymbol{\xi})$ be functions such that $\varphi \in \mathrm{C}_{0}^{\infty}(B(\mathbf{z}, R))$ and $\eta \in \mathrm{C}_{0}^{\infty}\left(B\left(\boldsymbol{\mu}, R_{1}\right)\right)$ with some $\mathbf{z}, \boldsymbol{\mu} \in \mathbb{R}^{d}$, and some fixed $R, R_{1}>0$. Then

$$
\lim _{\alpha \rightarrow \infty}\left[\frac{1}{\alpha^{d-1} \log \alpha} \operatorname{tr}\left(\varphi \operatorname{Op}_{\alpha}(\eta) D_{\alpha}\left(\chi_{\Omega}, \Lambda ; g_{p}\right)\right)-U\left(g_{p}\right) \mathfrak{V}_{1}(\varphi \eta ; \partial \Lambda, \partial \Omega)\right]=0 .
$$

The convergence is uniform

(1) in the domain $\Lambda$ in the sense specified in Remark 7.2,

(2) in the functions $h$ and $\eta$ in the sense that it is uniform in the functions $\varphi$, $\eta$ satisfying the bounds $\mathrm{N}^{(k)}(\varphi ; R), \mathrm{N}^{(k)}\left(\eta ; R_{1}\right) \lesssim 1$ for all $k=0,1,2, \ldots$, with some fixed constants.

This proposition follows from [Sob13, Theorem 11.1].

The next Proposition makes a statement similar to (7.2), but uniform in the radius $R$.

Lemma 7.4. Suppose that $\Lambda$ and $\Omega$ are as in Proposition 7.3. Let $\varphi \in \mathrm{C}_{0}^{\infty}(B(\mathbf{z}, R))$ with some $R \leq 1$. Then

$$
\lim _{\alpha R \rightarrow \infty} R^{1-d}\left[\frac{1}{\alpha^{d-1} \log (\alpha R)} \operatorname{tr}\left(\varphi D_{\alpha}\left(\chi_{\Omega}, \Lambda ; g_{p}\right)\right)-U\left(g_{p}\right) \mathfrak{V}_{1}(\varphi ; \partial \Lambda, \partial \Omega)\right]=0 .
$$

The convergence is uniform in the domain $\Lambda$ and the function $\varphi$ as specified in Proposition 7.3.

Proof. First we note that (7.2) with an arbitrary $\eta \in \mathrm{C}_{0}^{\infty}\left(\mathbb{R}^{d}\right)$, implies (7.2) with $\eta \equiv 1$. Indeed, Let $\eta \in \mathrm{C}_{0}^{\infty}$ be such that $\eta \chi_{\Omega}=\chi_{\Omega}$. Write:

$$
\varphi \mathrm{Op}_{\alpha}(\eta) \chi_{\Lambda} \mathrm{Op}_{\alpha}\left(\chi_{\Omega}\right)=\left[\varphi \mathrm{Op}_{\alpha}(\eta), \chi_{\Lambda}\right] \mathrm{Op}_{\alpha}\left(\chi_{\Omega}\right)+\varphi \chi_{\Lambda} \mathrm{Op}_{\alpha}\left(\chi_{\Omega}\right),
$$

so that

$$
\begin{array}{r}
\varphi \mathrm{Op}_{\alpha}(\eta)\left(\chi_{\Lambda} \mathrm{Op}_{\alpha}\left(\chi_{\Omega}\right) \chi_{\Lambda}\right)^{p}-\varphi\left(\chi_{\Lambda} \mathrm{Op}_{\alpha}\left(\chi_{\Omega}\right) \chi_{\Lambda}\right)^{p} \\
=\left[\varphi \mathrm{Op}_{\alpha}(\eta), \chi_{\Lambda}\right] \mathrm{Op}_{\alpha}\left(\chi_{\Omega}\right)\left(\chi_{\Lambda} \mathrm{Op}_{\alpha}\left(\chi_{\Omega}\right) \chi_{\Lambda}\right)^{p-1},
\end{array}
$$

for any $p=1,2, \ldots$ Therefore

$$
\left\|\varphi \mathrm{Op}_{\alpha}(\eta) D_{\alpha}\left(\chi_{\Omega}, \Lambda ; g_{p}\right)-\varphi D_{\alpha}\left(\chi_{\Omega}, \Lambda ; g_{p}\right)\right\|_{1} \leq 2\left\|\left[\varphi \mathrm{Op}_{\alpha}(\eta), \chi_{\Lambda}\right]\right\|_{1} .
$$


By (5.4), the right-hand side does not exceed $\mathrm{N}^{(d+2)}(\varphi ; R)(\alpha R)^{d-1}$, uniformly in the domain $\Lambda$. Therefore (7.2) leads to (7.2) with $\eta \equiv 1$, as claimed.

By rescaling, the operator in (7.3) is unitarily equivalent to

$$
\tilde{\varphi} D_{\nu}\left(\chi_{\Omega}, \tilde{\Lambda} ; g_{p}\right), \nu=\alpha R \rightarrow \infty,
$$

with

$$
\tilde{\varphi}(\mathbf{x})=\varphi(R \mathbf{x}), \tilde{\Lambda}=\Gamma(\tilde{\Phi}), \tilde{\Phi}(\hat{\mathbf{x}})=R^{-1} \Phi(R \hat{\mathbf{x}}) .
$$

Clearly, $\tilde{\varphi} \in \mathrm{C}_{0}^{\infty}\left(R^{-1} \mathbf{z}, 1\right)$, and $\mathbf{N}^{(k)}(\tilde{\varphi} ; 1) \lesssim 1$ for all $k=0,1,2, \ldots$ Furthermore, $M_{\tilde{\Phi}}=M_{\Phi}$, and, because of the restriction $R \leq 1$ the functions $\nabla \Phi$ and $\nabla \tilde{\Phi}$ satisfy (7.1) with the same modulus of continuity $\varepsilon(r)$. Thus one can use formula (7.2) with $\eta \equiv 1$ :

$$
\lim _{\nu \rightarrow \infty}\left[\frac{1}{\nu^{d-1} \log \nu} \operatorname{tr}\left(\tilde{\varphi} D_{\nu}\left(\chi_{\Omega}, \tilde{\Lambda} ; g_{p}\right)\right)-U\left(g_{p}\right) \mathfrak{V}_{1}(\tilde{\varphi} ; \partial \tilde{\Lambda}, \partial \Omega)\right]=0 .
$$

Observing that

$$
\mathfrak{V}_{1}(\tilde{\varphi} ; \partial \tilde{\Lambda}, \partial \Omega)=R^{1-d} \mathfrak{V}_{1}(\varphi ; \partial \Lambda, \partial \Omega),
$$

we get (7.3), as claimed.

\section{Proof of Theorem 2.7 for $\alpha T \lesssim 1$}

In this section we begin the proof of Theorem 2.7.

8.1 Localization estimates for the operator $D_{\alpha}(a, \Lambda ; f)$. Using Lemma 4.5, here we convert the bounds obtained previously in Sect. 6 into appropriate bounds for the operator (1.2). In Lemmas 8.1 - 8.3 we assume that $f \in C_{0}^{n}(\mathbb{R})$ with $n \geq 3$. Unless otherwise stated, the constants in the estimates below do not depend on the function $f$ or parameters $\alpha, \ell, T$.

LEMMA 8.1. Let the symbol $a$ be either $a_{T}=a_{T, \Omega}$ or $\chi_{\Omega}$ with a bounded Lipschitz region $\Omega$. Suppose that the sets $\Lambda$, $\Pi$ satisfy

$$
B(\mathbf{z}, 2 \ell) \cap \Lambda=B(\mathbf{z}, 2 \ell) \cap \Pi .
$$

Suppose also that $\alpha \ell \gtrsim 1,0<T \lesssim 1$. Then

$$
\left\|\chi_{B(\mathbf{z}, \ell)}\left(D_{\alpha}(a, \Lambda ; f)-D_{\alpha}(a, \Pi ; f)\right)\right\|_{1} \lesssim N_{n}(f)(\alpha \ell)^{d-1} .
$$

If $\Pi=\mathbb{R}^{d}$, then

$$
\left\|\chi_{B(\mathbf{z}, \ell)} D_{\alpha}(a, \Lambda ; f)\right\|_{1} \lesssim N_{n}(f)(\alpha \ell)^{d-1} .
$$

The constants in (8.2) and (8.3) do not depend on the sets $\Lambda$ and $\Pi$.

If, in addition, $\Pi$ is a Lipschitz region, then

$$
\left\|\chi_{B(\mathbf{z}, \ell)} D_{\alpha}\left(a_{T}, \Lambda ; f\right)\right\|_{1} \lesssim N_{n}(f)(\alpha \ell)^{d-1} \log \left(\min \left\{\alpha \ell, \frac{1}{T}\right\}+1\right) .
$$

If $\Pi$ is a basic Lipschitz domain, than the constant in (8.4) is uniform in $\Pi$. 
Proof. The bound (8.3) is a direct consequence of $(8.2)$, since $D_{\alpha}\left(a, \mathbb{R}^{d} ; f\right)=0$.

To prove (8.2) let $\varphi \in \mathrm{C}_{0}^{\infty}$ be a function such that $\varphi(\mathbf{x})=1, \mathbf{x} \in B(\mathbf{z}, \ell), \varphi(\mathbf{x})=0$ for $\mathbf{x} \notin B(\mathbf{z}, 2 \ell)$, and $\ell^{m}\left|\nabla^{m} \varphi\right| \lesssim 1$ for all $m=1,2, \ldots$ Since $\chi_{B(\mathbf{z}, \ell)} \varphi=\varphi$, in view of (8.1), the relation (4.13) is satisfied, and hence we can use Lemma 4.5 with $\tilde{b}=b=a$. It follows from (5.6) or (6.16) that

$$
\left\|\left[\varphi, \mathrm{Op}_{\alpha}(a)\right]\right\|_{1} \lesssim(\alpha \ell)^{d-1} .
$$

Now (4.15) leads to (8.2).

Proof of (8.4). We use the same function $\varphi$ as above. By (8.2) we may assume that $\Lambda=\Pi$. Due to (4.14), the left-hand side of (8.4) does not exceed

$$
N_{n}(f)\left(\left\|\varphi \chi_{\Pi} \mathrm{Op}_{\alpha}\left(a_{T}\right)\left(I-\chi_{\Pi}\right)\right\|_{1}+\left\|\left[\varphi, \mathrm{Op}_{\alpha}\left(a_{T}\right)\right]\right\|_{1}\right) .
$$

It remains to apply (6.16) and (6.17).

Lemma 8.2. Let the sets $\Lambda$ and $\Pi$ satisfy (8.1). Suppose that $\Omega$ is a bounded Lipschitz region. Let $\alpha \ell \gtrsim 1$ and $\alpha \ell T \lesssim 1$. Then

$$
\left\|\chi_{B(\mathbf{z}, \ell)}\left(D_{\alpha}\left(a_{T}, \Lambda ; f\right)-D_{\alpha}\left(\chi_{\Omega}, \Pi ; f\right)\right)\right\|_{1} \lesssim N_{n}(f)(\alpha \ell)^{d-1} .
$$

The constant in (8.5) is independent of the sets $\Lambda$ and $\Pi$.

Proof. We use Lemma 4.5 with $b=a_{T}, \tilde{b}=\chi_{\Omega}$, with the function $\varphi$ defined in the proof of the previous lemma. By (6.16),

$$
\left\|\left[\mathrm{Op}_{\alpha}\left(a_{T}\right), \varphi\right]\right\|_{1} \lesssim(\alpha \ell)^{d-1} .
$$

Furthermore, by Corollary 6.5,

$$
\left\|\varphi \mathrm{Op}_{\alpha}\left(a_{T}-\chi_{\Omega}\right)\right\|_{1} \lesssim(\alpha \ell)^{d-1} .
$$

Now (4.15) leads to (8.5).

Lemma 8.3. Suppose that $\Omega$ is a bounded Lipschitz region. Suppose that $\mathbb{R}^{d} \backslash \Lambda \subset$ $B\left(\mathbf{0}, R_{0}\right)$ with some $R_{0}>0$, and let $\varphi \in C^{\infty}\left(\mathbb{R}^{d}\right)$ be a bounded function such that $\varphi(\mathbf{x})=0$ for $\mathbf{x} \in B\left(\mathbf{0}, R_{0}\right)$ and $\varphi(\xi)=1$ for $|\mathbf{x}|>2 R_{0}$. Let $\alpha \gtrsim 1,0<T \lesssim 1$. Then

$$
\left\|\varphi D_{\alpha}\left(a_{T}, \Lambda ; f\right)\right\|_{1} \lesssim N_{n}(f) \alpha^{d-1} .
$$

The constant in (8.6) is independent of $\Lambda$, but may depend on $R_{0}$ and $\varphi$.

Proof. We use Lemma 4.5 with $\Pi=\mathbb{R}^{d}$, so that $\Lambda$ and $\Pi$ satisfy (4.13). Thus we can use the bound (4.16) with $b=a_{T}$, and hence the left-hand side of (8.6) is bounded from above by

$$
\left\|\left[\varphi, \mathrm{Op}_{\alpha}\left(a_{T}\right)\right]\right\|_{1}=\left\|\left[I-\varphi, \mathrm{Op}_{\alpha}\left(a_{T}\right)\right]\right\|_{1} .
$$

The function $1-\varphi$ is compactly supported. Now using (6.16) we arrive at (8.6). 
8.2 Proof of Theorem 2.7 for $\boldsymbol{\alpha} \boldsymbol{T} \lesssim \mathbf{1}$. We assume that $\Lambda$ and $\Omega$ satisfy conditions of Theorem 2.7, and that $\Omega$ is bounded (see Remark 2.8). For brevity, in the proof we often use the short-hand notation $D_{\alpha}(f)=D_{\alpha}\left(a_{T}, \Lambda ; f\right)$ and $\mathfrak{V}_{1}=$ $\mathfrak{V}_{1}(1 ; \partial \Lambda, \partial \Omega)$. As in the previous section, we use the notation $g_{p}(t)=t^{p}, p=$ $1,2, \ldots$

REMARK 8.4. It is clear that we can use for the operator $D_{\alpha}(f)$ all the bounds, established earlier, with arbitrary smooth functions $f$ without the assumption that $f$ is compactly supported. Indeed, since $\left\|a_{T}\right\| \lesssim 1$ we have $f\left(a_{T}\right)=f\left(a_{T}\right) \zeta\left(a_{T}\right)$ and $f\left(W_{\alpha}\right)=f\left(W_{\alpha}\right) \zeta\left(W_{\alpha}\right), W_{\alpha}=W_{\alpha}\left(a_{T} ; \Lambda\right)$, with some fixed $\zeta \in \mathrm{C}_{0}^{\infty}(\mathbb{R})$. In particular, this observation applies to the polynomial functions $g_{p}$.

We precede the proof with some bounds for the integral (2.12), see [Sob, Lemma 4.6]:

Proposition 8.5. If $f \in \mathrm{W}^{1, \infty}(\mathbb{R})$, then

$$
|U(f)| \leq 2\left\|f^{\prime}\right\|_{L^{\infty}} .
$$

If $f$ satisfies (2.2) with $n=1$ and some $0<R \leq 1$, then

$$
|U(f)| \lesssim R^{\frac{\gamma}{2}}|f|_{1}
$$

Now we can proceed with the proof of Theorem 2.7. It follows the idea of [Sob], and consists of three parts: first we consider polynomial functions $f$, then extend it to arbitrary $\mathrm{C}^{2}$-functions, and finally complete the proof for functions satisfying the conditions of the Theorem.

Step 1. Polynomial $f$. Let $f=g_{p}$. Let $R_{0}$ be such that either $\Lambda \subset B\left(\mathbf{0}, R_{0}\right)$ or $\mathbb{R}^{d} \backslash \overline{\Lambda \subset B\left(\mathbf{0}, R_{0}\right) \text {. Let } \varphi} \in \mathrm{C}_{0}^{\infty}\left(\mathbb{R}^{d}\right)$ be a function such that $\varphi(\mathbf{x})=1$ for $|\mathbf{x}| \leq R_{0}$, and $\varphi(\mathbf{x})=0$ for $|\mathbf{x}|>2 R_{0}$. Since $\alpha R_{0} \gtrsim 1$ and $\alpha R_{0} T \lesssim 1$, from Lemma 8.2 we obtain that

$$
\left\|\varphi D_{\alpha}\left(a_{T}, \Lambda ; g_{p}\right)-\varphi D_{\alpha}\left(\chi_{\Omega}, \Lambda ; g_{p}\right)\right\|_{1} \lesssim\left(\alpha R_{0}\right)^{d-1},
$$

with an implicit constant depending on $p$. In combination with Proposition 7.1 this gives the equality

$$
\lim _{\substack{\alpha \rightarrow \infty \\ \alpha T \lesssim 1}} \frac{1}{\alpha^{d-1} \log \alpha} \operatorname{tr}\left(\varphi D_{\alpha}\left(a_{T}, \Lambda ; g_{p}\right)\right)=U\left(g_{p}\right) \mathfrak{V}_{1} .
$$

If $\Lambda \subset B\left(\mathbf{0}, R_{0}\right)$, then $\varphi \chi_{\Lambda}=\chi_{\Lambda}$, and hence these asymptotics coincide with the sought formula (2.15). If $\mathbb{R}^{d} \backslash \Lambda \subset B\left(\mathbf{0}, R_{0}\right)$, then we invoke Lemma 8.3 which implies that

$$
\lim _{\substack{\alpha \rightarrow \infty \\ \alpha T \lesssim 1}} \frac{1}{\alpha^{d-1} \log \alpha} \operatorname{tr}\left((I-\varphi) D_{\alpha}\left(a_{T}, \Lambda ; g_{p}\right)\right)=0 .
$$

Together with (8.9) this gives (2.15) for $f=g_{p}$ again. 
Step 2. Arbitrary functions $f \in C^{2}(\mathbb{R})$. The extension from polynomials to more general functions is done in the same way as in [Sob], and we remind this argument for the sake of completeness.

Let $\zeta \in \mathrm{C}_{0}^{\infty}(\mathbb{R})$ be the function introduced before Proposition 8.5. Let $g$ be a polynomial such that

$$
\|(f-g) \zeta\|_{C^{2}}<\delta .
$$

For $g$ we can use the formula (2.15) established previously:

$$
\lim _{\substack{\alpha \rightarrow \infty \\ \alpha T \lesssim 1}} \frac{1}{\alpha^{d-1} \log \alpha} \operatorname{tr} D_{\alpha}(g)=U(g) \mathfrak{V}_{1} .
$$

On the other hand, by virtue of (6.18), we have

$$
\left\|D_{\alpha}(f-g)\right\|_{1}=\left\|D_{\alpha}((f-g) \zeta)\right\|_{1} \lesssim\|(f-g) \zeta\|_{C^{2}} \alpha^{d-1} \log \alpha \lesssim \delta \alpha^{d-1} \log \alpha,
$$

for $\alpha T \lesssim 1$, and also, by (8.7),

$$
|U(f)-U(g)|=|U(f-g)|=|U((f-g) \zeta)| \leq 2\left\|((f-g) \zeta)^{\prime}\right\|_{L^{\infty}<2 \delta .}
$$

Thus, using (8.10) and the additivity

$$
D_{\alpha}(f)=D_{\alpha}(g)+D_{\alpha}(f-g), U(f)=U(g)+U(f-g),
$$

we get

$$
\limsup _{\substack{\alpha \rightarrow \infty \\ \alpha T \lesssim 1}}\left|\frac{1}{\alpha^{d-1} \log \alpha} \operatorname{tr} D_{\alpha}(f)-U(f) \mathfrak{V}_{1}\right| \lesssim \delta .
$$

Since $\delta>0$ is arbitrary, we obtain (2.15) for arbitrary $f \in C^{2}(\mathbb{R})$.

Step 3. Completion of the proof. Let $f$ be a function as specified in Theorem 2.7. Without loss of generality suppose that the set $X$ consists of one point, and this point is $z=0$.

Let $\zeta \in \mathrm{C}_{0}^{\infty}(\mathbb{R})$ be a real-valued function, such that $\zeta(t)=1$ for $|t| \leq 1 / 2$. Represent $f=f_{R}^{(1)}+f_{R}^{(2)}, 0<R \leq 1$, where $f_{R}^{(1)}(t)=f(t) \zeta\left(t R^{-1}\right), f_{R}^{(2)}(t)=$ $f(t)-f_{R}^{(1)}(t)$. It is clear that $f_{R}^{(2)} \in C^{2}(\mathbb{R})$, so one can use the formula $(2.15)$ established in Step 2 of the proof:

$$
\lim _{\substack{\alpha \rightarrow \infty \\ \alpha T \lesssim 1}} \frac{1}{\alpha^{d-1} \log \alpha} D_{\alpha}\left(f_{R}^{(2)}\right)=U\left(f_{R}^{(2)}\right) \mathfrak{V}_{1} .
$$

For $f_{R}^{(1)}$ we use Theorem 2.6 taking into account that $\left|f_{R}^{(1)}\right|_{2} \lesssim|f|_{2}$ :

$$
\left|\operatorname{tr} D_{\alpha}\left(f_{R}^{(1)}\right)\right| \lesssim R^{\gamma-\sigma}|f|_{2} \alpha^{d-1} \log \alpha, \quad \alpha \gtrsim 1, \alpha T \lesssim 1
$$


for any $\sigma \in\left(d \beta^{-1}, \gamma\right), \sigma \in(0,1]$. Moreover, by (8.8),

$$
\left|U\left(f_{R}^{(1)}\right)\right| \lesssim R^{\frac{\gamma}{2}}|f|_{1} .
$$

Thus, using (8.11) and the additivity

$$
D_{\alpha}(f)=D_{\alpha}\left(f_{R}^{(2)}\right)+D_{\alpha}\left(f_{R}^{(1)}\right), U(f)=U\left(f_{R}^{(2)}\right)+U\left(f_{R}^{(1)}\right),
$$

we get the bound

$$
\limsup _{\alpha \rightarrow \infty}\left|\frac{1}{\alpha^{d-1} \log \alpha} D_{\alpha}(f)-U(f) \mathfrak{V}_{1}\right| \lesssim|f|_{2}\left(R^{\gamma-\sigma}+R^{\frac{\gamma}{2}}\right) .
$$

Since $R$ is arbitrary, by taking $R \rightarrow 0$, we obtain (2.15) for the function $f$.

\section{Proof of Theorem 2.7 for $\alpha T \gtrsim 1$}

9.1 Proof of Theorem 2.7: basic smooth domains $\Lambda$. We begin with an asymptotic formula for the trace

$$
\operatorname{tr}\left(\varphi D_{\alpha}\left(a_{T}, \Lambda ; g_{p}\right)\right)
$$

with a basic $C^{1}$-domain $\Lambda$, a function $\varphi \in C_{0}^{\infty}\left(\mathbb{R}^{d}\right)$, and a polynomial $g_{p}(t)=t^{p}$, $p=1,2, \ldots$. As before we assume that $\Omega$ is bounded. We assume that $f \in C_{0}^{n}(\mathbb{R})$ with some $n \geq 3$. Our immediate objective is to prove the following result.

Theorem 9.1. Let $\Lambda$ be a basic $C^{1}$-domain, and let $\Omega$ be a bounded $\mathrm{C}^{3}$-region. Suppose that $\alpha T \gtrsim 1$, and that $\varphi \in \mathrm{C}_{0}^{\infty}\left(\mathbb{R}^{d}\right)$. Then

$$
\lim _{\substack{T \rightarrow 0 \\ \alpha T \gtrsim 1}} \frac{1}{\alpha^{d-1} \log \frac{1}{T}} \operatorname{tr}\left(\varphi D_{\alpha}\left(a_{T}, \Lambda ; g_{p}\right)\right)=U\left(g_{p}\right) \mathfrak{V}_{1}(\varphi ; \partial \Lambda, \partial \Omega) .
$$

By rescaling and translating we may assume that in Theorem 9.1 the support of $\varphi$ is contained in the ball $B(\mathbf{0}, 1)$. We also assume that

$$
\mathrm{N}^{(d+2)}(\varphi ; 1) \leq 1 \text {. }
$$

If the support of $\varphi$ has an empty intersection with the boundary $\partial \Lambda$, then by (8.3),

$$
\left|\operatorname{tr}\left(\varphi D_{\alpha}\left(a_{T}, \Lambda ; f\right)\right)\right| \lesssim N_{n}(f) \alpha^{d-1},
$$

and hence (9.1) automatically holds.

It remains to consider the case where $\operatorname{supp} \varphi \cap \partial \Lambda \neq \varnothing$. For this case we construct a convenient partition of unity. For $\Lambda=\Gamma(\Phi)$ let $\mathbf{x}_{\hat{\mathbf{n}}}=(\ell \hat{\mathbf{n}}, \Phi(\ell \hat{\mathbf{n}})), \hat{\mathbf{n}} \in \mathbb{Z}^{d-1}$, $\ell=(\alpha T)^{-1}$, be the points on the boundary $\partial \Lambda$. Then the balls $B\left(\mathbf{x}_{\hat{\mathbf{n}}}, r\right), \hat{\mathbf{n}} \in \mathbb{Z}^{d-1}$, with $r=\ell \sqrt{(1+M)^{2}+1}$ form a covering of the strip

$$
\Lambda_{\ell}=\left\{\mathbf{x} \in \mathbb{R}^{d}: \Phi(\hat{\mathbf{x}})<x_{d}<\Phi(\hat{\mathbf{x}})+\ell\right\} \subset \Lambda .
$$


Let $\phi_{\hat{\mathbf{n}}} \in \mathrm{C}_{0}^{\infty}\left(\mathbb{R}^{d}\right)$ be a partition of unity subordinate to this covering. We may assume that

$$
\mathrm{N}^{(n)}\left(\phi_{\hat{\mathbf{n}}} ; \ell\right) \lesssim 1, n=0,1,2, \ldots,
$$

uniformly in $\hat{\mathbf{n}} \in \mathbb{Z}^{d-1}$. Denote

$$
w_{1}(\mathbf{x})=\varphi(\mathbf{x}) \sum_{\hat{\mathbf{n}} \in \mathbb{Z}^{d-1}} \phi_{\hat{\mathbf{n}}}(\mathbf{x}), w_{2}(\mathbf{x})=\varphi-w_{1}(\mathbf{x}) .
$$

Note that

$$
\#\left\{\hat{\mathbf{n}} \in \mathbb{Z}^{d-1}: \mathbf{x}_{\hat{\mathbf{n}}} \in \operatorname{supp} \varphi\right\} \lesssim \ell^{1-d} .
$$

Lemma 9.2. Let $\Lambda$ be a basic Lipschitz domain, and let $\Omega$ be a bounded Lipschitz region. Let $0<T \lesssim 1, \alpha T \gtrsim 1$. Then

$$
\left|\operatorname{tr}\left[w_{1}\left(D_{\alpha}\left(a_{T}, \Lambda ; f\right)-D_{\alpha}\left(\chi_{\Omega}, \Lambda ; f\right)\right)\right]\right| \lesssim N_{n}(f) \alpha^{d-1},
$$

uniformly in $\Lambda$.

Proof. The trace on the left-hand side of (9.7) coincides with

$$
\sum_{\hat{\mathbf{n}}} \operatorname{tr}\left[\varphi \phi_{\hat{\mathbf{n}}}\left(D_{\alpha}\left(a_{T}, \Lambda ; f\right)-D_{\alpha}\left(\chi_{\Omega}, \Lambda ; f\right)\right)\right]
$$

The support of $\phi_{\hat{\mathbf{n}}}$ is contained in $B\left(\mathbf{x}_{\hat{\mathbf{n}}}, r\right), r \asymp \ell$, and also $\alpha \ell=T^{-1} \gtrsim 1, \alpha \ell T=1$. Thus from Lemma 8.2 for each summand we obtain the bound by

$$
N_{n}(f) \mathrm{N}^{(d+2)}\left(\varphi \phi_{\hat{\mathbf{n}}} ; \ell\right)(\alpha \ell)^{d-1} .
$$

In view of (9.2), (9.4) and (9.6), this leads to (9.7).

Lemma 9.3. Let $\Lambda$ be a basic Lipschitz domain, and let $\Omega$ be a bounded Lipschitz region. Suppose that $0<T \lesssim 1, \alpha T \gtrsim 1$. Then

$$
\left|\operatorname{tr}\left(w_{2} D_{\alpha}\left(a_{T}, \Lambda ; f\right)\right)\right| \lesssim N_{n}(f) \alpha^{d-1}
$$

uniformly in $\Lambda$.

Proof. Since the function $w_{2}$ satisfies (4.13) with $\Pi=\mathbb{R}^{d}$, the bound (4.16) implies that the left-hand side is bounded (up to the factor $N_{n}(f)$ ) by

$$
\left\|\left[w_{2}, \mathrm{Op}_{\alpha}\left(a_{T}\right)\right]\right\|_{1} \leq\left\|\left[\varphi, \mathrm{Op}_{\alpha}\left(a_{T}\right)\right]\right\|_{1}+\sum_{\hat{\mathbf{n}}}\left\|\left[\varphi \phi_{\hat{\mathbf{n}}}, \mathrm{Op}_{\alpha}\left(a_{T}\right)\right]\right\|_{1}
$$

By (6.16),

$$
\begin{aligned}
\left\|\left[\varphi, \mathrm{Op}_{\alpha}\left(a_{T}\right)\right]\right\|_{1} & \lesssim \mathrm{N}^{(d+2)}(\varphi ; 1) \alpha^{d-1}, \\
\left\|\left[\varphi \phi_{\hat{\mathbf{n}}}, \mathrm{Op}_{\alpha}\left(a_{T}\right)\right]\right\|_{1} & \lesssim \mathrm{N}^{(d+2)}\left(\varphi \phi_{\hat{\mathbf{n}}} ; \ell\right)(\alpha \ell)^{d-1} .
\end{aligned}
$$

As in the proof of Lemma 9.2, the number of summands does not exceed $\ell^{1-d}$, so that the right-hand side of $(9.9)$ is bounded by $N_{n}(f) \alpha^{d-1}$, as claimed. 
Proof of Theorem 9.1. By Lemmas 9.2, 9.3 and Remark 8.4,

$$
\begin{aligned}
& \left|\operatorname{tr}\left(\varphi D_{\alpha}\left(a_{T}, \Lambda ; g_{p}\right)\right)-\operatorname{tr}\left(w_{1} D_{\alpha}\left(\chi_{\Omega}, \Lambda ; g_{p}\right)\right)\right| \\
& \quad=\left|\operatorname{tr}\left(w_{2} D_{\alpha}\left(a_{T}, \Lambda ; g_{p}\right)\right)\right| \lesssim \alpha^{d-1}
\end{aligned}
$$

with an implicit constant depending on $p$. Thus it suffices to find the asymptotics of the required form for the second term on the left-hand side. To analyse the asymptotics for each term in the definition of $w_{1}$ ( see (9.5)) we use the formula (7.3) with the function $\phi_{\hat{\mathbf{n}}} \varphi$ and $R=\ell$. Since $\ell=(\alpha T)^{-1}$, and $\alpha \ell=T^{-1}$ the formula (7.3) rewrites as follows:

$$
\begin{aligned}
& \lim _{\substack{T \rightarrow 0, \alpha T \gtrsim 1}} \ell^{1-d}\left[\frac{1}{\alpha^{d-1} \log \frac{1}{T}} \operatorname{tr}\left(\phi_{\hat{\mathbf{n}}} \varphi D_{\alpha}\left(\chi_{\Omega}, \Lambda ; g_{p}\right)\right)\right. \\
& \left.\quad-U\left(g_{p}\right) \mathfrak{V}_{1}\left(\phi_{\hat{\mathbf{n}}} \varphi ; \partial \Lambda, \partial \Omega\right)\right]=0,
\end{aligned}
$$

uniformly in $\hat{\mathbf{n}} \in \mathbb{Z}^{d-1}$. Therefore

$$
\begin{aligned}
& \lim \sup \left|\frac{1}{\alpha^{d-1} \log \frac{1}{T}} \operatorname{tr}\left(w_{1} D_{\alpha}\left(\chi_{\Omega}, \Lambda ; g_{p}\right)\right)-U\left(g_{p}\right) \mathfrak{V}_{1}\left(w_{1} ; \partial \Lambda, \partial \Omega\right)\right| \\
& \quad \leq \lim \sup \sum_{\hat{\mathbf{n}}}\left|\frac{1}{\alpha^{d-1} \log \frac{1}{T}} \operatorname{tr}\left(\phi_{\hat{\mathbf{n}}} \varphi D_{\alpha}\left(\chi_{\Omega}, \Lambda ; g_{p}\right)\right)-U\left(g_{p}\right) \mathfrak{V}_{1}\left(\phi_{\hat{\mathbf{n}}} \varphi ; \partial \Lambda, \partial \Omega\right)\right| \\
& \quad \lesssim \lim \sup \ell^{1-d} \max _{\hat{\mathbf{n}}}\left|\frac{1}{\alpha^{d-1} \log \frac{1}{T}} \operatorname{tr}\left(\phi_{\hat{\mathbf{n}}} \varphi D_{\alpha}\left(\chi_{\Omega}, \Lambda ; g_{p}\right)\right)-U\left(g_{p}\right) \mathfrak{V}_{1}\left(\phi_{\hat{\mathbf{n}}} \varphi ; \partial \Lambda, \partial \Omega\right)\right|
\end{aligned}
$$

as $T \rightarrow 0, \alpha T \gtrsim 1$. Here we have used (9.6) again. Given the uniformity in $\hat{\mathbf{n}} \in \mathbb{Z}^{d-1}$ and the bound (9.6), the formula (9.11) implies that

$$
\lim _{T \rightarrow 0}\left[\frac{1}{\alpha^{d-1} \log \frac{1}{T}} \operatorname{tr}\left(w_{1} D_{\alpha}\left(\chi_{\Omega}, \Lambda ; g_{p}\right)\right)-U\left(g_{p}\right) \mathfrak{V}_{1}\left(w_{1} ; \partial \Lambda, \partial \Omega\right)\right]=0 .
$$

Taking into account (9.10) and that $w_{1}(\mathbf{x})=\varphi(\mathbf{x})$ for $\mathbf{x} \in \partial \Lambda$, this leads to (9.1).

9.2 Proof of Theorem 2.7: basic piece-wise smooth domains $\Lambda$. Here we extend the formula (9.1) to piece-wise smooth domains $\Lambda$. Our argument follows the proof of [Sob15, Theorem 4.1]. For simplicity we assume that only $\Lambda$ is piecewise smooth, whereas $\Omega$ remains smooth. This simplification preserves the idea of [Sob15], but allows one to avoid some routine technical work that would have been just a modified repetition of the proof from [Sob15].

Theorem 9.4. Let $\Lambda$ be a basic piece-wise $C^{1}$-domain, and let $\Omega$ be a bounded $\mathrm{C}^{3}$-region. Suppose that $\varphi \in \mathrm{C}_{0}^{\infty}\left(\mathbb{R}^{d}\right)$. Then the formula (9.1) holds. 
Proof. Assume as before that $\varphi(\mathbf{x})=0$ for $|\mathbf{x}|>1$ and (9.2) holds. We follow the idea of the proof of [Sob15, Theorem 4.1]. Cover the ball $B(\mathbf{0}, 1)$ with open balls of radius $\varepsilon>0$, such that the number of intersections is bounded from above uniformly in $\varepsilon$. Introduce a subordinate partition of unity $\left\{\phi_{j}\right\}, j=1,2, \ldots$, such that $\mathrm{N}^{(n)}\left(\phi_{j} ; \varepsilon\right) \lesssim 1$ uniformly in $j=1,2, \ldots$ By (9.3) contributions to (9.1) from the balls having empty intersection with $\partial \Lambda$, equal zero.

Let $\Sigma$ be the set of indices such that the ball indexed by $j \in \Sigma$ has a non-empty intersection with the set $(\partial \Lambda)_{\mathrm{S}}$, see Subsect. 2.2 for the definition. Since the set $(\partial \Lambda)_{\mathrm{S}}$ is built out of $(d-2)$-dimensional Lipschitz surfaces, we have

$$
\# \Sigma \lesssim \varepsilon^{2-d}
$$

By (8.4), for each ball we have the bound

$$
\left|\operatorname{tr}\left(\varphi \phi_{j} D_{\alpha}\left(a_{T}, \Lambda ; g_{p}\right)\right)\right| \lesssim(\alpha \varepsilon)^{d-1} \log \frac{1}{T}
$$

with an implicit constant depending on $p$, uniformly in $j=1,2, \ldots$, if $\alpha \varepsilon \gtrsim 1$. By virtue of (9.12), this implies that

$$
\sum_{j \in \Sigma}\left|\operatorname{tr}\left(\varphi \phi_{j} D_{\alpha}\left(a_{T}, \Lambda ; g_{p}\right)\right)\right| \lesssim \varepsilon \alpha^{d-1} \log \frac{1}{T}, \text { if } \alpha \varepsilon \gtrsim 1
$$

As

$$
\sum_{j \in \Sigma}\left|\mathfrak{V}_{1}\left(\varphi \phi_{j}, \partial \Lambda ; \partial \Omega\right)\right| \lesssim \varepsilon
$$

(see (2.11) for the definition of $\mathfrak{V}_{1}$ ), we can rewrite the last two formulas as follows:

$$
\limsup _{\substack{T \rightarrow 0 \\ \alpha T \gtrsim 1}} \sum_{j \in \Sigma}\left|\frac{1}{\alpha^{d-1} \log \frac{1}{T}} \operatorname{tr}\left(\varphi \phi_{j} D_{\alpha}\left(a_{T}, \Lambda ; g_{p}\right)\right)-U\left(g_{p}\right) \mathfrak{V}_{1}\left(\varphi \phi_{j}, \partial \Lambda, \partial \Omega\right)\right| \lesssim \varepsilon .
$$

Let us now turn to the balls with indices $j \notin \Sigma$. We may assume that they are separated from $(\partial \Lambda)_{\mathrm{s}}$. Thus in each such ball the boundary of $\Lambda$ is $\mathrm{C}^{1}$. By (8.2), we may assume that the entire $\Lambda$ is $C^{1}$, and hence Theorem 9.1 is applicable. Together with (9.13), this gives

$$
\limsup _{\substack{T \rightarrow 0 \\ \alpha T \gtrsim 1}} \mid \frac{1}{\alpha^{d-1} \log \frac{1}{T}} \operatorname{tr}\left(\varphi D_{\alpha}\left(a_{T}, \Lambda ; g_{p}\right)-U\left(g_{p}\right) \mathfrak{V}_{1}(\varphi, \partial \Lambda, \partial \Omega) \mid \lesssim \varepsilon .\right.
$$

Since $\varepsilon>0$ is arbitrary, this proves the Theorem. 
9.3 Proof of Theorem 2.7: completion. Theorem 9.4 extends to arbitrary piece-wise $C^{1}$ region $\Lambda$ by using the standard partition of unity argument based on Lemma 8.1. Also, as mentioned earlier, one can extend Theorem 9.4 to the piece-wise $\mathrm{C}^{3}$-regions $\Omega$. We omit this argument since it repeats the proofs in [Sob15].

The extension of Theorem 9.4 to arbitrary functions $f$ specified in Theorem 2.7, is done in the same way as in the proof of Theorem 2.7 for $\alpha T \lesssim 1$, with the help of the bounds (2.10) and (6.18). We omit the details.

\section{Comparison with known asymptotic formulas}

10.1 Coefficient $\mathcal{B}_{\boldsymbol{d}}$. The asymptotics of $\operatorname{tr} D_{\alpha}(a, \Lambda ; f)$ for a fixed symbol $a$ as $\alpha \rightarrow \infty$ have been studied rather extensively in the 1980's by H. Widom and R. Roccaforte, e.g. [Wid85] and references therein. It is interesting and instructive to compare there findings with the asymptotics in Theorem 2.7. As shown in [Wid85, BB91], for a fixed smooth symbol $a$, smooth $f$ and smooth $\Lambda$ one can write out a complete asymptotic expansion in powers of $\alpha^{-1}$. In this section we focus only on the first coefficient of this expansion that we denoted by $\mathcal{B}_{d}(a ; \partial \Lambda, f)$ in the Introduction, see (1.4). It has a more complicated form than the coefficient $\mathfrak{V}_{1}$ defined in (2.11) and featuring in Theorem 2.7, and it is described below.

For a function $f: \mathbb{C} \rightarrow \mathbb{C}$ and any $s_{1}, s_{2} \in \mathbb{C}$ define the integral

$$
U\left(s_{1}, s_{2} ; f\right)=\int_{0}^{1} \frac{f\left((1-t) s_{1}+t s_{2}\right)-\left[(1-t) f\left(s_{1}\right)+t f\left(s_{2}\right)\right]}{t(1-t)} d t .
$$

The integral $U(f)$ defined in $(2.12)$ is easily expressed as $U(f)=U(1,0 ; f)$. It is clear that $U\left(s_{1}, s_{2} ; 1\right)=U\left(s_{1}, s_{2} ; t\right)=0$, for all $s_{1}, s_{2} \in \mathbb{C}$. This integral is finite for functions $f \in C^{0, \varkappa}(\mathbb{C}), \varkappa \in(0,1]$. It is also Hölder-continuous: for any $\delta \in(0, \varkappa)$ we have

$$
\left|U\left(s_{1}, s_{2} ; f\right)-U\left(r_{1}, r_{2} ; f\right)\right| \lesssim \operatorname{Lip}_{\varkappa}(f)\left(\left|s_{1}-r_{1}\right|^{\delta}+\left|s_{2}-r_{2}\right|^{\delta}\right)
$$

where

$$
\operatorname{Lip}_{\varkappa}(f)=\sup _{z \neq w} \frac{|f(z)-f(w)|}{|z-w|^{\varkappa}} .
$$

Note also that

$$
U\left(s_{1}, s_{1} ; f\right)=0, \quad U\left(s_{1}, s_{2} ; f\right)=U\left(s_{2}, s_{1} ; f\right), \forall s_{1}, s_{2} \in \mathbb{C} .
$$

For a symbol $a=a(\xi), \xi \in \mathbb{R}$ define

$$
\mathcal{B}(a ; f):=\frac{1}{8 \pi^{2}} \lim _{\varepsilon \rightarrow 0} \iint_{\left|\xi_{1}-\xi_{2}\right|>\varepsilon} \frac{U\left(a\left(\xi_{1}\right), a\left(\xi_{2}\right) ; f\right)}{\left|\xi_{1}-\xi_{2}\right|^{2}} d \xi_{1} d \xi_{2} .
$$


If $f$ is smooth, then this definition coincides with the standard double integral. In particular, if $f^{\prime \prime}$ is bounded, then

$$
|\mathcal{B}(a ; f)| \lesssim\left\|f^{\prime \prime}\right\|_{\mathrm{L}^{\infty}} \iint \frac{\left|a\left(\xi_{1}\right)-a\left(\xi_{2}\right)\right|^{2}}{\left|\xi_{1}-\xi_{2}\right|^{2}} d \xi_{1} d \xi_{2} .
$$

This estimate was first pointed out in [Wid82a]. Note that $\mathcal{B}(a ; f)$ is invariant under the change $a(\xi) \rightarrow a(\tau \xi)$ with an arbitrary $\tau>0$. If $f$ is allowed to be non-smooth, as in Condition 2.1, then the finiteness of the limit in (10.4) is not trivial, and we comment on this later, in Proposition 10.2.

As shown in [Wid82a], see also [LSS16b], in the case $d=1$, for smooth $f$ and $a$ we have $\operatorname{tr} D_{\alpha}\left(a, \mathbb{R}_{+} ; f\right) \rightarrow \mathcal{B}(a ; f)$ as $\alpha \rightarrow \infty$. For the multi-dimensional case the asymptotic coefficient $\mathcal{B}_{d}(a ; \partial \Lambda, f)$ is defined as follows. For a unit vector $\mathbf{e} \in$ $\mathbb{R}^{d}, d \geq 2$, introduce the hyperplane

$$
\Pi_{\mathbf{e}}:=\left\{\boldsymbol{\xi} \in \mathbb{R}^{d}: \mathbf{e} \cdot \boldsymbol{\xi}=0\right\} .
$$

Introduce the orthogonal coordinates $\boldsymbol{\xi}=(\stackrel{\circ}{\boldsymbol{\xi}}, t)$ such that $\stackrel{\circ}{\boldsymbol{\xi}} \in \Pi_{\mathbf{e}}$ and $t \in \mathbb{R}$. Then we set

$$
\begin{aligned}
\mathcal{B}_{d}(a ; \partial \Lambda, f) & :=\frac{1}{(2 \pi)^{d-1}} \int_{\partial \Lambda} \mathcal{A}_{d}\left(a, \mathbf{n}_{\mathbf{x}} ; f\right) d S_{\mathbf{x}}, \quad \mathcal{A}_{d}(a, \mathbf{e} ; f) \\
& :=\int_{\Pi_{\mathbf{e}}} \mathcal{B}(a(\stackrel{\circ}{\boldsymbol{\xi}}, \cdot) ; f) d \stackrel{\circ}{\boldsymbol{\xi}} .
\end{aligned}
$$

For the smooth symbol $a$ and smooth function $f$ it was proved by H. Widom (see [Wid80] and [Wid85]), that the trace of $D_{\alpha}(a, \Lambda ; f)$ satisfies (1.4). Clearly, the formula (1.4) describes the asymptotics of $\operatorname{tr} D_{\alpha}$ for the symbol $a=a_{T}$, as $\alpha \rightarrow \infty$ and $T>0$ is fixed. On the other hand, Theorem 2.7 offers an asymptotic formula in two parameters: $\alpha \rightarrow \infty$ and $T \rightarrow 0$. Our aim now is to compare the asymptotic coefficient defined in (2.11), with the coefficient $\mathcal{B}_{d}\left(a_{T}, \partial \Lambda ; f\right)$ as $T \rightarrow 0$. The relevant calculations are quite involved, and to avoid further complications, we assume that $\Omega$ is smooth. In fact, for these purposes it will be sufficient to assume that $\Omega$ is $C^{2}$-smooth.

Theorem 10.1. Let $\Lambda$ satisfy Condition 2.4, and let $\Omega \subset \mathbb{R}^{d}$ be a bounded $C^{2}$ region.

Let the function $f$ satisfy Condition 2.1 with $n=2$ and some $\gamma>0$.

Let $a=a_{T}$ be the symbol defined in Subsection 2.3 with some $\beta>\max \left\{d \gamma^{-1}, d\right\}$. Then

$$
\lim _{T \rightarrow 0} \frac{1}{\log \frac{1}{T}} \mathcal{B}_{d}\left(a_{T} ; \partial \Lambda, f\right)=U(f) \mathfrak{V}_{1}(1 ; \partial \Lambda, \partial \Omega) .
$$

As pointed out in the Introduction, due to this theorem, the formula (2.14) can be rewritten in the form (1.9), and hence it can be viewed as an extension of (1.4) to the asymptotics in two parameters, $\alpha$ and $T$. Such an asymptotic formula was obtained in [LSS16b] for the one-dimensional case. Note that (2.15) cannot be rewritten in the same way. 
10.2 Coefficient $\mathcal{B}_{\boldsymbol{d}}$ for the symbol $\boldsymbol{a}_{\boldsymbol{T}}$. We begin our analysis of the coefficient $\mathcal{B}_{d}(a ; \partial \Lambda, f)$ with studying the multi-scale symbols introduced in Subsection 5.3. Let the symbol $a$ satisfy (5.13) with the scale $\tau$ and amplitude $v$ that satisfy (5.16) and (5.17) respectively. Assume that (5.14) holds. As the region $\Lambda$ is always fixed, for brevity we omit $\partial \Lambda$ from the notation and write simply $\mathcal{B}_{d}(a ; f)$.

From now on we assume that $f$ satisfies Condition 2.1 with some $\gamma>0$ and $n=2$. We use the notation $\varkappa=\min \{1, \gamma\}$. The next proposition is borrowed from [Sob16, Theorem 6.1].

Proposition 10.2. Suppose that $f$ satisfies Condition 2.1 with $n=2, \gamma>0$ and some $R>0$. Let the symbol $a \in \mathrm{C}^{\infty}(\mathbb{R})$ be a real-valued symbol described above. Then for any $\sigma \in(0, \varkappa]$ we have

$$
|\mathcal{B}(a ; f)| \lesssim|f|{ }_{2} R^{\gamma-\sigma} V_{\sigma, 1}(v, \tau),
$$

with a constant independent of $f$, uniformly in the functions $\tau, v$, and the symbol $a$.

We note another useful result from [Sob16]. It describes the contribution of "close" points $\xi_{1}$ and $\xi_{2}$ in the coefficient (10.4). For $r>0$ define

$$
\begin{aligned}
& \mathcal{B}^{(1)}(a ; f ; r)=\frac{1}{8 \pi^{2}} \lim _{\varepsilon \rightarrow 0} \iint_{\varepsilon<\left|\xi_{1}-\xi_{2}\right|<r} \frac{U\left(a\left(\xi_{1}\right), a\left(\xi_{2}\right) ; f\right)}{\left|\xi_{1}-\xi_{2}\right|^{2}} d \xi_{1} d \xi_{2}, \\
& \mathcal{B}^{(2)}(a ; f ; r)=\frac{1}{8 \pi^{2}} \iint_{\left|\xi_{1}-\xi_{2}\right| \geq r} \frac{U\left(a\left(\xi_{1}\right), a\left(\xi_{2}\right) ; f\right)}{\left|\xi_{1}-\xi_{2}\right|^{2}} d \xi_{1} d \xi_{2} .
\end{aligned}
$$

The integral (10.8) is estimated in the following proposition.

Proposition 10.3. Suppose that $f$ satisfies Condition 2.1 with $n=2, \gamma>0$ and some $R>0$. Let $a \in C^{\infty}(\mathbb{R})$ be as above. Suppose also that $r \leq \tau_{\text {inf }} / 2$. Then for any $\delta \in[0, \varkappa)$, the following bound holds:

$$
\left|\mathcal{B}^{(1)}(a ; f ; r)\right| \lesssim|f|{ }_{2} R^{\gamma-\varkappa} r^{\delta} V_{\varkappa, 1+\delta}(v, \tau),
$$

uniformly in the functions $\tau, v$, and the symbol $a$.

This bound follows from [Sob16, Corollary 6.5].

For the case $d \geq 2$, using the notations (10.8) and (10.9) define

$$
\mathcal{A}_{d}^{(k)}(a, \mathbf{e} ; f ; r):=\int_{\Pi_{\mathbf{e}}} \mathcal{B}^{(k)}(a(\stackrel{\circ}{\boldsymbol{\xi}}, \cdot) ; f ; r) d \stackrel{\circ}{\boldsymbol{\xi}}, \quad k=1,2 .
$$

We can estimate the quantities $\mathcal{A}_{d}$ and $\mathcal{A}_{d}^{(1)}$ similarly to (10.7) and (10.10):

$$
\left|\mathcal{A}_{d}(a, \mathbf{e} ; f)\right| \lesssim|f|_{2} V_{\varkappa, 1}(v, \tau)
$$


and

$$
\left|\mathcal{A}_{d}^{(1)}(a, \mathbf{e} ; f ; r)\right| \lesssim|f|{ }_{2} r^{\delta} V_{\varkappa, 1+\delta}(v, \tau), \forall \delta \in[0, \varkappa), r \leq \frac{\tau_{\text {inf }}}{2},
$$

uniformly in $\tau, v, a$ and $\mathbf{e} \in \mathbb{S}^{d-1}$. Indeed, the symbol $b(t):=a(\stackrel{\circ}{\boldsymbol{\xi}}, t)$ satisfies conditions (5.13) and (5.17) with the amplitude $v_{\mathbf{e}}(t)=v(\stackrel{\circ}{\boldsymbol{\xi}}, t)$ and the scaling function $\tau_{\mathbf{e}}(t)=\tau(\stackrel{\circ}{\xi}, t)$. By $(10.7)$,

$$
|\mathcal{B}(b ; f)| \lesssim|f|_{2} V_{\varkappa, 1}\left(v_{\mathbf{e}}, \tau_{\mathbf{e}}\right),
$$

uniformly in e, and in the functions $v, \tau$. Integrating over $\stackrel{\circ}{\boldsymbol{\xi}}$ we get (10.12).

Furthermore, since $\inf \tau_{\mathbf{e}} \geq \tau_{\text {inf }}$, from (10.10) we get that

$$
\left|\mathcal{B}^{(1)}(b ; f ; r)\right| \lesssim|f|_{2} r^{\delta} V_{\varkappa, 1+\delta}\left(v_{\mathbf{e}}, \tau_{\mathbf{e}}\right), \forall r \leq \frac{\tau_{\text {inf }}}{2},
$$

for any $\delta \in[0, \varkappa)$, uniformly in $\mathbf{e}, v, \tau$, as above. Integrating over $\stackrel{\circ}{\boldsymbol{\xi}}$, we get (10.13).

As we have pointed out previously, in view of (6.5), the symbol $a_{T}$ satisfies (5.13) with the functions $v$ and $\tau$ defined in (6.6). Recall also that $\tau_{\text {inf }} \asymp T$. Together with (6.9) and (6.10), the estimates (10.12) and (10.13) yield the bounds

$$
\left|\mathcal{A}_{d}(a, \mathbf{e} ; f)\right| \lesssim|f|_{2} \log \frac{1}{T}
$$

and

$$
\left|\mathcal{A}_{d}^{(1)}(a, \mathbf{e} ; f ; r)\right| \lesssim|f|_{2}, \forall r \leq \frac{\tau_{\text {inf }}}{2},
$$

uniformly in $\mathbf{e} \in \mathbb{S}^{d-1}$. From now on we take $r=\theta T$ where $\theta>0$ is chosen to satisfy $r \leq \frac{\tau_{\text {inf }}}{2}$. The parameter $\theta$ is fixed, and we are not concerned about the dependence of the forthcoming estimates on $\theta$.

Let us now take care of the integral $\mathcal{A}_{d}^{(2)}$.

Lemma 10.4. Let $r=\theta T$, with a $\theta>0$ described above. Then for all $0<T \lesssim 1$ we have

$$
\left|\mathcal{A}_{d}^{(2)}\left(a_{T}, \mathbf{e} ; f ; \theta T\right)-\mathcal{A}_{d}^{(2)}\left(\chi_{\Omega}, \mathbf{e} ; f ; \theta T\right)\right| \lesssim|f|_{1},
$$

uniformly in $\mathbf{e} \in \mathbb{S}^{d-1}$.

Proof. By definitions (10.9) and (10.11), the bounds (2.3) and (10.2) imply that

$$
\begin{aligned}
\left|\mathcal{A}_{d}^{(2)}\left(a_{T}, \mathbf{e} ; f ; \theta T\right)-\mathcal{A}_{d}^{(2)}\left(\chi_{\Omega}, \mathbf{e} ; f ; \theta T\right)\right| & \lesssim|f|_{1} \int_{\mathbb{R}^{d-1}} \iiint_{|t-s|>\theta T} \frac{\mid a_{T}(\stackrel{\circ}{\boldsymbol{\xi}}, t)-\chi_{\Omega}\left(\stackrel{\circ}{\boldsymbol{\xi}, t},\left.t\right|^{\delta}\right.}{|s-t|^{2}} d s d t d \dot{\xi} \\
& \lesssim T^{-1}|f|_{1} \int\left|a_{T}(\boldsymbol{\xi})-\chi_{\Omega}(\boldsymbol{\xi})\right|^{\delta} d \boldsymbol{\xi}
\end{aligned}
$$

for any $\delta \in(0, \varkappa)$. By $(6.7)$, for $\delta \in\left(d \beta^{-1}, \varkappa\right)$ the integral on the right-hand side is finite and it does not exceed $T$, whence (10.16). 


\section{Coefficient $\mathcal{A}_{d}^{(2)}\left(\chi_{\Omega}, \mathrm{e} ; f ; \theta T\right)$}

11.1 Smooth surfaces. In order to study the integral $\mathcal{A}_{d}^{(2)}$ we need to investigate the following model problem. For $\rho>0$, let

$$
\mathrm{C}_{\rho}^{(n)}:=(-2 \rho, 2 \rho)^{n}
$$

be the $n$-dimensional cube. We use two ways of labelling the coordinates:

$$
\begin{aligned}
\boldsymbol{\xi} & =\left(\hat{\boldsymbol{\xi}}, \xi_{d}\right), \quad \hat{\boldsymbol{\xi}}:=\left(\xi_{1}, \xi_{2}, \ldots, \xi_{d-1}\right), \\
\boldsymbol{\xi} & =\left(\stackrel{\circ}{\boldsymbol{\xi}}, \xi_{l}\right), \stackrel{\circ}{\boldsymbol{\xi}}:=\left(\xi_{1}, \ldots, \xi_{l-1}, \xi_{l+1}, \ldots \xi_{d}\right),
\end{aligned}
$$

where $l=1,2, \ldots, d$. If $l=d$, then, clearly, $\hat{\boldsymbol{\xi}}=\stackrel{\circ}{\boldsymbol{\xi}}$. Let $\Psi \in \mathrm{C}^{2}\left(\overline{\mathrm{C}_{\rho}^{(d-1)}}\right)$ be a function with values in the interval $(-2 \rho, 2 \rho)$. We focus on the surface

$$
S=S(\Psi):=\left\{\boldsymbol{\xi} \in \mathcal{C}_{\rho}^{(d)}: \xi_{d}=\Psi(\hat{\boldsymbol{\xi}})\right\}
$$

For each $\stackrel{\circ}{\xi} \in \mathcal{C}_{\rho}^{(d-1)}$ define the set

$$
\mathrm{X}_{l}(\stackrel{\circ}{\boldsymbol{\xi}}):=\mathbf{X}_{l}(\stackrel{\circ}{\boldsymbol{\xi}} ; \Psi):=\left\{\xi_{l} \in(-2 \rho, 2 \rho):\left(\stackrel{\circ}{\boldsymbol{\xi}}, \xi_{l}\right) \in S\right\} .
$$

Let us record some useful facts about the set $\mathrm{X}_{l}(\stackrel{\circ}{\boldsymbol{\xi}})$ :

LEMMA 11.1. Let $\mathbf{e}_{l}$ be the unit basis vector along the direction $l$, and let $\mathbf{n}_{\boldsymbol{\xi}}$ be a unit normal to $S$ at the point $\boldsymbol{\xi} \in S$. For any function $u$, continuous on the cube $\overline{\mathrm{e}_{\rho}^{(d)}}$ and any $l=1,2, \ldots, d$ we have

$$
\int_{\mathcal{C}_{\rho}^{(d-1)}} \sum_{\boldsymbol{\xi}: \xi_{l} \in \mathrm{X}_{l}(\stackrel{\circ}{\boldsymbol{\xi}})} u(\boldsymbol{\xi}) d \dot{\xi}=\int_{S} u(\boldsymbol{\xi})\left|\mathbf{n}_{\boldsymbol{\xi}} \cdot \mathbf{e}_{l}\right| d S_{\boldsymbol{\xi}}
$$

In particular, the function $\#\left(\mathrm{X}_{l}(\stackrel{\bullet}{\xi})\right)$ counting the number of elements is finite for a.e. $\stackrel{\circ}{\xi} \in \mathcal{C}_{\rho}^{(d-1)}$ and

$$
\int_{\mathcal{C}_{\rho}^{(d-1)}} \#\left(\mathrm{X}_{l}(\stackrel{\circ}{\boldsymbol{\xi}})\right) d \stackrel{\circ}{\boldsymbol{\xi}}=\int_{S}\left|\mathbf{n}_{\boldsymbol{\xi}} \cdot \mathbf{e}_{l}\right| d S_{\boldsymbol{\xi}}
$$

Proof. Equality (11.5) follows from (11.4) with $u \equiv 1$.

Let us prove now (11.4). We denote by $\Xi: \mathcal{C}_{\rho}^{(d-1)} \rightarrow \mathcal{C}_{\rho}^{(d-1)}$ the mapping

$$
\Xi(\hat{\boldsymbol{\xi}}):=\left(\xi_{1}, \ldots, \xi_{l-1}, \xi_{l+1}, \ldots, \xi_{d-1}, \Psi(\hat{\boldsymbol{\xi}})\right) .
$$


Then by the Change of Variables Formula (see, e.g., [EG92, Theorem 2, p. 99]), for any continuous function $u$ on the cube $\overline{\mathrm{C}_{\rho}^{(d)}}$, we have

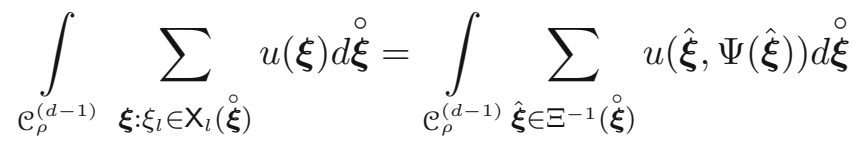

$$
\begin{aligned}
& =\int_{\mathcal{C}_{\rho}^{(d-1)}} u(\hat{\boldsymbol{\xi}}, \Psi(\hat{\boldsymbol{\xi}})) J_{\Xi}(\hat{\boldsymbol{\xi}}) d \hat{\boldsymbol{\xi}}
\end{aligned}
$$

where $J_{\Xi}$ is the Jacobian of the map $\Xi$. A direct calculation shows that

$$
J_{\Xi}=\left|\partial_{\xi_{l}} \Psi\right|=\frac{\left|\partial_{\xi_{l}} \Psi\right|}{\sqrt{1+|\nabla \Psi|^{2}}} \sqrt{1+|\nabla \Psi|^{2}}=\left|\mathbf{n}_{\boldsymbol{\xi}} \cdot \mathbf{e}_{l}\right| \sqrt{1+|\nabla \Psi|^{2}} .
$$

Thus the equality (11.6) becomes (11.4).

11.2 Function $\boldsymbol{m}$. For $\stackrel{\circ}{\boldsymbol{\xi}} \in \mathcal{C}_{\rho}^{(d-1)}$ define the following function $m=m(\stackrel{\circ}{\xi})$ : if $\mathrm{X}_{l}(\stackrel{\circ}{\xi})=\varnothing$, then we define

$$
m(\stackrel{\circ}{\xi}):=(4 \rho)^{-1}
$$

If $\#\left(\mathrm{X}_{l}(\stackrel{\circ}{\boldsymbol{\xi}})\right)=N \geq 1$, then we label the points $\xi \in \mathrm{X}_{l}(\stackrel{\circ}{\boldsymbol{\xi}})$ in increasing order: $\xi^{(1)}<\xi^{(2)}<\ldots<\xi^{(N)}$, and define

$$
m(\stackrel{\circ}{\boldsymbol{\xi}}):=\sum_{j=1}^{N} \rho_{j}(\stackrel{\circ}{\boldsymbol{\xi}})^{-1}, \quad\left\{\begin{array}{l}
\rho_{j}(\stackrel{\circ}{\boldsymbol{\xi}}):=\operatorname{dist}\left\{\xi^{(j)}, \mathrm{X}_{l}(\stackrel{\circ}{\boldsymbol{\xi}}) \backslash\left\{\xi^{(j)}\right\}\right\}, N \geq 2 \\
\rho_{1}:=4 \rho, N=1
\end{array}\right.
$$

The function $m$ is well-defined since $\#\left(\mathrm{X}_{l}(\stackrel{\circ}{\boldsymbol{\xi}})\right)<\infty$ for a.e. $\stackrel{\circ}{\boldsymbol{\xi}} \in \mathcal{C}_{\rho}^{(d-1)}$.

In all the bounds obtained below the constants are independent of the function $\Psi \subset C^{2}$ and parameter $\rho>0$. We begin with an estimate for $m(\stackrel{\circ}{\xi})$, see [Wid90, p. 185] or [Sob13, Chapter 13].

Proposition 11.2. Let $\Psi \in \mathrm{C}^{2}\left(\overline{\left.\mathrm{e}_{\rho}^{(d-1)}\right)}, d \geq 2\right.$, with some $\rho>0$. Then the function $m$ satisfies the bound

$$
\int_{\mathcal{C}_{\rho}^{(d-1)}} m(\stackrel{\circ}{\boldsymbol{\xi}}) d \stackrel{\circ}{\boldsymbol{\xi}} \lesssim \rho^{d-2}\left(1+\rho\left\|\nabla^{2} \Psi\right\|_{\mathrm{L}^{\infty}}\right)
$$

Here is a useful consequence of this proposition: 
Lemma 11.3. Let the function $\Psi$ be as in Proposition 11.2. Let $\rho_{j}(\stackrel{\circ}{\boldsymbol{\xi}})$ be the distances defined in (11.8) for the set $\mathbf{X}_{l}(\stackrel{\circ}{\boldsymbol{\xi}})$. Let $\rho_{0}(\stackrel{\circ}{\boldsymbol{\xi}})=\inf _{j} \rho_{j}(\stackrel{\circ}{\boldsymbol{\xi}})$ if $\#\left(\mathbf{X}_{l}(\stackrel{\circ}{\boldsymbol{\xi}})\right) \geq 1$, and $\rho_{0}(\stackrel{\circ}{\boldsymbol{\xi}})=4 \rho$, if $\mathbf{X}_{l}(\stackrel{\circ}{\boldsymbol{\xi}})=\varnothing$. For all $\varepsilon \in(0,4 \rho)$ denote

$$
M_{\varepsilon}:=M_{\varepsilon}(\Psi):=\left\{\stackrel{\circ}{\boldsymbol{\xi}} \in \mathcal{C}_{\rho}^{(d-1)}: \rho_{0}(\stackrel{\circ}{\boldsymbol{\xi}})<\varepsilon\right\}
$$

Then

$$
\left|M_{\varepsilon}\right| \lesssim \varepsilon \rho^{d-2}\left(1+\rho\left\|\nabla^{2} \Psi\right\|_{L^{\infty}}\right) .
$$

Proof. By the definition (11.7), (11.8),

$$
m(\stackrel{\circ}{\boldsymbol{\xi}}) \geq \rho_{0}(\stackrel{\circ}{\boldsymbol{\xi}})^{-1}
$$

so by virtue of Chebyshev's inequality, we get from (11.9) that

$$
\varepsilon^{-1} \int_{M_{\varepsilon}} d \stackrel{\circ}{\boldsymbol{\xi}} \leq \int_{M_{\varepsilon}} m(\stackrel{\circ}{\boldsymbol{\xi}}) d \stackrel{\circ}{\mathrm{\xi}} \leq C \rho^{d-2}\left(1+\rho\left\|\nabla^{2} \Psi\right\|_{\mathrm{L}^{\infty}}\right) .
$$

This leads to (11.11).

It immediately follows from the above lemma that

$$
\#\left(X_{l}(\stackrel{\circ}{\boldsymbol{\xi}})\right)<\infty, \quad \stackrel{\circ}{\boldsymbol{\xi}} \notin M(\Psi) \equiv \bigcap_{\varepsilon>0} M_{\varepsilon}(\Psi), \quad \text { and }|M(\Psi)|=\lim _{\varepsilon \rightarrow 0}\left|M_{\varepsilon}(\Psi)\right|=0 .
$$

11.3 Asymptotics of $\mathcal{A}_{d}^{(2)}\left(\chi_{\Omega}, \mathrm{e} ; \boldsymbol{f} ; \boldsymbol{\theta T}\right)$. In order to use the conclusions of Lemma 11.3, we adopt the following conventions.

- We always assume that the Cartesian coordinates in $\mathbb{R}^{d}$ are chosen in such a way that the unit vector $\mathbf{e}$ coincides with the basis vector $\mathbf{e}_{l}$, so that the vector $\stackrel{\xi}{\xi}$ featuring in (10.5) is given by (11.1).

- In each set $D_{j}$ of the covering (6.3), we re-label the remaining coordinates to ensure that the part of the surface $\partial \Omega$ inside $D_{j}$ is given by the equality $\xi_{d}=\Psi_{j}(\hat{\boldsymbol{\xi}})$. The coordinates $\hat{\boldsymbol{\xi}}$ and $\stackrel{\circ}{\boldsymbol{\xi}}$ do not necessarily coincide, and the choice of $\hat{\boldsymbol{\xi}}$ may be different in different $D_{j}$ 's.

Denote by $\left\{\phi_{j}\right\}, \tilde{\phi}$ a partition of unity subordinate to the covering (6.3). Thus we split $\mathcal{A}_{d}^{(2)}\left(\chi_{\Omega}, \mathbf{e} ; f ; \theta T\right)$ into the sum

$$
\mathcal{A}_{d}^{(2)}\left(\chi_{\Omega}, \mathbf{e} ; f ; \theta T\right)=\sum_{j} \mathcal{K}_{j}(T)+\tilde{\mathcal{K}}(T),
$$


where

$$
\mathcal{K}_{j}(T):=\frac{1}{8 \pi^{2}} \int_{\mathbb{R}^{d-1}} \iint_{\theta T<|t-s|} \phi_{j}(\stackrel{\circ}{\boldsymbol{\xi}}, t) \frac{U\left(\chi_{\Omega}(\stackrel{\circ}{\boldsymbol{\xi}}, t), \chi_{\Omega}(\stackrel{\circ}{\boldsymbol{\xi}}, s) ; f\right)}{|t-s|^{2}} d s d t d \dot{\circ}
$$

and the integral $\tilde{\mathcal{K}}(T)$ is defined in a similar way with the function $\tilde{\phi}$.

The properties $U(0,0 ; f)=U(1,1 ; f)=0$ and $U(1,0 ; f)=U(0,1 ; f)$ allow one to rewrite $\mathcal{K}_{j}(T), \tilde{\mathcal{K}}(T)$ in the form

$$
\mathcal{K}_{j}(T)=\frac{U(1,0 ; f)}{4 \pi^{2}} \int_{\mathbb{R}^{d-1}} \mathcal{S}_{j}(\stackrel{\circ}{\boldsymbol{\xi}} ; T) d \stackrel{\circ}{\boldsymbol{\xi}}, \tilde{\mathcal{K}}(T)=\frac{U(1,0 ; f)}{4 \pi^{2}} \int_{\mathbb{R}^{d-1}} \tilde{\mathcal{S}}(\stackrel{\circ}{\boldsymbol{\xi}} ; T) d \boldsymbol{\xi}
$$

where

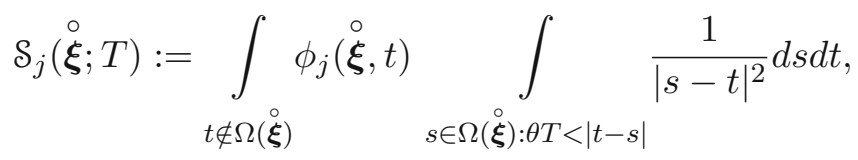

with

$$
\Omega(\stackrel{\circ}{\xi}):=\{t \in \mathbb{R}:(\stackrel{\circ}{\xi}, t) \in \Omega\}
$$

and $\tilde{\mathcal{S}}(\stackrel{\circ}{\xi} ; T)$ is defined in a similar way with the function $\tilde{\phi}$. Since the set $\tilde{D}$ is separated from the surface $S$, the integral $\tilde{\mathcal{S}}(\stackrel{\circ}{\xi} ; T)$ is taken over the set where $|s| \lesssim 1$, $|s-t| \gtrsim 1$, and therefore

$$
\tilde{\mathcal{K}}(T) \lesssim 1
$$

for all $T>0$. Let us now analyse $\mathcal{S}_{j}(\stackrel{\circ}{\xi} ; T)$. First we quote the following elementary statement, proved in [LSS16b, Lemma 9.4].

Proposition 11.4. Let $J_{k}=\left(s_{k}, t_{k}\right) \subset \mathbb{R}, k=1,2, \ldots, N$ be a finite collection of open intervals, such that their closures are pairwise disjoint, and let $J=\cup_{k} J_{k}$. Suppose that $0<T \lesssim 1$ and $\left|J_{k}\right| \leq d_{1}, k=1,2, \ldots, N$, with some $d_{1}>0$. Then

$$
\sum_{k=1}^{N} \int_{t \notin J} \int_{|t-s| \geq T, s \in J_{k}} \frac{1}{|t-s|^{2}} d s d t \lesssim N \log \left(\frac{1}{T}+1\right)
$$

with a constant depending only on $d_{1}$.

Assume in addition that

$$
\left|J_{k}\right| \geq d_{0}, k=1,2, \ldots, N, \min _{j \neq k} \operatorname{dist}\left\{J_{k}, J_{j}\right\} \geq d_{0} .
$$


with some $d_{0} \in\left(0, d_{1}\right]$. Let $\varphi \in \mathrm{C}(\mathbb{R}) \cap \mathrm{L}^{\infty}(\mathbb{R})$ be a function. Then

$$
\begin{aligned}
& \sum_{k=1}^{N} \int_{t \notin J} \varphi(t) \int_{|t-s| \geq T, s \in J_{k}} \frac{1}{|t-s|^{2}} d s d t \\
& \quad=\log \frac{1}{T} \sum_{k=1}^{N}\left(\varphi\left(s_{k}\right)+\varphi\left(t_{k}\right)\right)+N\|\varphi\|_{\mathrm{L}^{\infty}} O(1), T \rightarrow 0,
\end{aligned}
$$

where $O(1)$ depends only on $d_{0}$ and $d_{1}$.

Now we can derive the following property of $\mathcal{S}_{j}(\stackrel{\circ}{\boldsymbol{\xi}} ; T)$.

Lemma 11.5. Let us fix a vector $\mathbf{e} \in \mathbb{S}^{d-1}$ and the index $j$. Let $S=S\left(\Psi_{j}\right)$ and $M=M\left(\Psi_{j}\right)$ be as defined in (11.2) and (11.12) respectively. Then for all $\stackrel{\circ}{\xi} \notin M$ we have

$$
\mathcal{S}_{j}(\stackrel{\circ}{\boldsymbol{\xi}} ; T)=\log \frac{1}{T} \sum_{\substack{\circ \\ \boldsymbol{\xi}:(\boldsymbol{\xi}, t) \in S}} \phi_{j}(\boldsymbol{\xi})+\#\left(\mathbf{X}_{l}\left(\stackrel{\circ}{\xi} ; \Psi_{j}\right)\right) O(1), T \rightarrow 0
$$

Moreover,

$$
\lim _{T \rightarrow 0} \frac{1}{\log \frac{1}{T}} \mathcal{K}_{j}(T)=\frac{U(1,0 ; f)}{4 \pi^{2}} \int_{S} \phi_{j}(\boldsymbol{\xi})\left|\mathbf{n}_{\boldsymbol{\xi}} \cdot \mathbf{e}\right| d S_{\boldsymbol{\xi}}
$$

Proof. Let $\rho>0$ be such that $D_{j} \subset \mathcal{C}_{\rho}^{(d)}$. By (11.12) the set

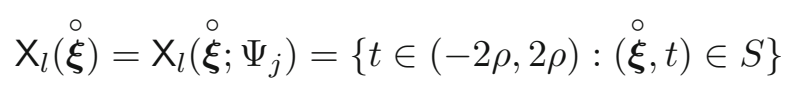

is finite for each $\stackrel{\circ}{\xi} \notin M$. Now the asymptotics (11.18) follow from (11.17). Furthermore, (11.16) implies that

$$
\left|\mathcal{S}_{j}(\stackrel{\circ}{\boldsymbol{\xi}} ; T)\right| \lesssim \#\left(\mathbf{X}_{l}(\stackrel{\circ}{\boldsymbol{\xi}})\right) \log \frac{1}{T}, \forall \stackrel{\circ}{\forall} \notin M .
$$

Thus, by the Dominated Convergence Theorem, (11.18) leads to the formula

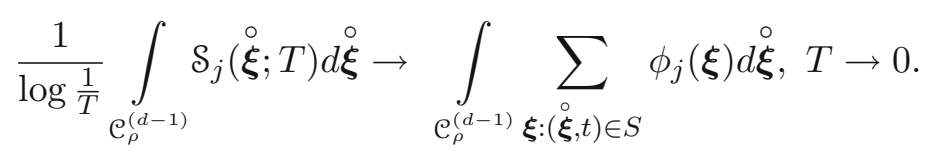

According to (11.4), the right-hand side coincides with

$$
\int_{S} \phi_{j}(\boldsymbol{\xi})\left|\mathbf{n}_{\boldsymbol{\xi}} \cdot \mathbf{e}\right| d S_{\boldsymbol{\xi}}
$$

and hence (11.19) holds. 
11.4 Proof of Theorem 10.1. Now we put together the formula (11.19) and the bounds (11.15), (10.16). This leads to the asymptotic formula

$$
\lim _{T \rightarrow 0} \frac{1}{\log \frac{1}{T}} \mathcal{A}_{d}^{(2)}\left(a_{T}, \mathbf{e} ; f ; \theta T\right)=\frac{U(f)}{4 \pi^{2}} \int_{\partial \Omega}\left|\mathbf{n}_{\boldsymbol{\xi}} \cdot \mathbf{e}\right| d S_{\boldsymbol{\xi}} .
$$

In view of the bound (10.15) the same formula formula holds for the coefficient $\mathcal{A}_{d}\left(a_{T}, \mathbf{e} ; f\right)$. On the other hand, this coefficient satisfies the bound (10.14) uniformly in $\mathbf{e} \in \mathbb{S}^{d-1}$. Therefore, by the Dominated Convergence Theorem, we get for the integral $\mathcal{B}_{d}($ see $(10.5))$ the asymptotics

$$
\lim _{T \rightarrow 0} \frac{1}{\log \frac{1}{T}} \mathcal{B}_{d}\left(a_{T} ; f\right)=\frac{U(f)}{(2 \pi)^{d+1}} \int_{\partial \Lambda} \int_{\partial \Omega}\left|\mathbf{n}_{\boldsymbol{\xi}} \cdot \mathbf{n}_{\mathbf{x}}\right| d S_{\boldsymbol{\xi}} d S_{\mathbf{x}},
$$

which coincides with the claimed formula (10.6).

\section{Acknowledgements}

The author is grateful to W. Spitzer for useful remarks. Thanks also go to the anonymous referee for a number of useful suggestions. This work was supported by EPSRC Grant EP/J016829/1.

Open Access This article is distributed under the terms of the Creative Commons Attribution 4.0 International License (http://creativecommons .org/licenses/by/4.0/), which permits unrestricted use, distribution, and reproduction in any medium, provided you give appropriate credit to the original author(s) and the source, provide a link to the Creative Commons license, and indicate if changes were made.

\section{References}

[Ber71] F.A. BEREZIN. Wick and anti-Wick operator symbols. Math. USSR. Sb., (4)15 (1971), 577-606. Translation of Mat. Sb. (N.S.) 86(1971), no. 4, 578-610 (128 in old numeration).

[BS77] M.Š. BiRman and M.Z. Solomyak. Estimates of singular numbers of integral operators. Uspekhi Mat. Nauk (1)32 (1977), 17-84, Engl. transl. in: Russian Math. Surveys 32(1977), no. 1, 15-89. 1987.

[BKS91] M.Sh. Birman, G.E. Karadzhov and M.Z. SolomyaK. Boundedness conditions and spectrum estimates for the operators $b(X) a(D)$ and their analogs. In: Estimates and Asymptotics for Discrete Spectra of Integral and Differential Equations (Leningrad, 1989-90), Adv. Soviet Math. 7. American Mathematical Society, Providence, RI (1991), pp. 85-106.

[BS87] M.Š. BIRMan and M.Z. Solomyak. Spectral Theory of Self-Adjoint Operators in Hilbert Space. Reidel, Dordrecht (1987).

[BB91] A.M. Budylin and V.S. Buslaev. On the asymptotic behaviour of the spectral characteristics of an integral operator with a difference kernel on expanding domains. In: Differential Equations, Spectral Theory, Wave Propagation (Russian), Vol. 305. Probl. Mat. Fiz., 13. Leningrad. Univ., Leningrad (1991), pp. 16-60. 
[Dav95] E.B. Davies. Spectral Theory and Differential Operators. CUP, Cambridge (1995).

[EG92] L.C. Evans and R.F. GariePy. Measure Theory and Fine Properties of Functions. CRC Press, Boca Raton (1992).

[FH98] M.E. Fisher and R.E. HARTwig. Toeplitz determinants, some applications, theorems and conjectures. Advances in Chemical Physics, 15 (1968), 333.

[GK69] I. GokhBerG and M. KREIN. Introduction to the Theory of Linear Non-selfadjoint Operators. AMS, Providence (1969).

[HS89] B. HELFFER and J. SJÖSTRAND. Équation de Schrödinger avec champ magnétique et équation de Harper (French) [The Schrödinger equation with magnetic field, and the Harper equation], Schrödinger operators (Sonderborg, 1988), Lecture Notes in Phys. 345. Springer, Berlin (1989), pp. 118-197.

[Kra11] I. KRAsovsky. Aspects of Toeplitz determinants. In: Boundaries and Spectra of Random Walks (D. Lenz, F. Sobieczky, W. Woss, editors), Progress in Probability. Birkhäuser, Basel (2011).

[LW80] H. LANDAU and H. Widom. Eigenvalue distribution of time and frequency limiting. Journal of Mathematical Analysis and Applications, 77 (1980), 469-481.

[LSS] H. Leschke, A.V. Sobolev and W.L. Spitzer. Scaling of Rényi entanglement entropies of the free Fermi-gas ground state: A rigorous proof. Physical Review Letters, 112, 160403.

[LSS16a] H. Leschke, A.V. Sobolev, and W. Spitzer. Area law for the entanglement entropy of the free Fermi gas at nonzero temperature. Journal of Physics A: Mathematical and Theoretical, (30)49 (2016).

[LSS16b] H. Leschke, A.V. Sobolev and W.L. SpItzer. Trace formulas for WienerHopf operators with applications to entropies of free fermionic equilibrium states. arXiv:1605.04429 (2016).

[Old15] J.P. OldFIELD. Two-term Szegő theorem for generalised anti-Wick operators. Journal of Spectral Theory, 5 (2015), 751-781.

[Pie78] A. Pietsch. Operator Ideals. Deutscher Verlag der Wissenschaften - Mathematics (1978).

[Rot67] S.Yu. Rotfeld. Remarks on the singular numbers of the sum of compact operators. Funkt Anal. i ego Pril., (3)1 (1967), 95-96.

[Shu01] M.A. Shubin. Pseudodifferential Operators and Spectral Theory, 2nd edn. Springer, Berlin (2001).

[Sim05] B. Simon. Trace ideals and their applications, 2nd edn, Mathematical Surveys and Monographs, 120. American Mathematical Society, Providence, RI (2005).

[Sob13] A.V. Sobolev. Pseudo-differential operators with discontinuous symbols: Widom's Conjecture. Memoirs of AMS, (1043)222 (2013).

[Sob14] A.V. Sobolev. On the Schatten-von Neumann properties of some pseudodifferential operators. Journal of Functional Analysis, 266 (2014), 5886-5911.

[Sob15] A.V. SobolEv. Wiener-Hopf operators in higher dimensions: The Widom conjecture for piece-wise smooth domains. Integral Equations and Operator Theory, (3) 81 (2015), 435-449.

[Sob] A.V. Sobolev. Functions of self-adjoint operators in ideals of compact operators. Journal of LMS, (1)95 (2017), 157-176.

[Sob16] A.V. Sobolev. On a coefficient in trace formulas for Wiener-Hopf operators. Journal of Spectral Theory, (4)6 (2016), 1021-1045.

[Wid80] H. Widom. Szegö's limit theorem: The higher-dimensional matrix case. Journal of Functional Analysis, 39 (1980), 182-198. 
[Wid82a] H. Widom. A trace formula for Wiener-Hopf operators. Journal of Operator Theory, 8 (1982), 279-298.

[Wid82b] H. WidOM. On a class of integral operators with discontinuous symbol, Toeplitz centennial (Tel Aviv, 1981), Operator Theory: Adv. Appl. 4. Birkhäuser, Basel, Boston, MA, (1982), pp. 477-500.

[Wid85] H. Widom. Asymptotic Expansions for Pseudodifferential Operators on Bounded Domains, Lecture Notes in Mathematics, V. 1152. Springer, Berlin (1985).

[Wid90] H. Widom. On a class of integral operators on a half-space with discontinuous symbol. Journal of Functional Analysis, (1)88 (1990), 166-193.

Alexander V. Sobolev, Department of Mathematics, University College London, Gower Street, London, WC1E 6BT, UK a.sobolev@ucl.ac.uk

Received: October 18, 2016 Accepted: January 23, 2017 